\title{
Diagnosis of Hearing Deficiency using EEG based AEP Signals: CWT and improved-VGG16 Pipeline
}

\author{
Md Nahidul Islam ${ }^{\text {Corresp., } 1}{ }^{\text {, Norizam Sulaiman }}{ }^{1}$, Fahmid Al Farid ${ }^{2}$, Jia Uddin ${ }^{3}$, Salem A. Alyami ${ }^{4}$, Mamunur Rashid ${ }^{1}$, \\ Anwar PP Abdul Majeed ${ }^{5,6}$, Mohammad Ali Moni ${ }^{\text {Corresp. } 7}$ \\ ${ }^{1}$ Faculty of Electrical and Electronics Engineering Technology, Universiti Malaysia Pahang, Pekan, Pahang, Malaysia \\ 2 Faculty of Computing and Informatics, Multimedia University, Cyberjaya 63000, Malaysia \\ 3 Technology Studies Department, Endicott College, Woosong university, Daejeon, South Korea \\ 4 Department of Mathematics and Statistics, Imam Mohammad Ibn Saud Islamic University, Riyadh, Saudi Arabia \\ 5 Innovative Manufacturing, Mechatronics and Sports Laboratory, Faculty of Manufacturing and Mechatronic Engineering Technology, Universiti Malaysia \\ Pahang, Pekan, Pahang, Malaysia \\ 6 Centre for Software Development \& Integrated Computing, Universiti Malaysia Pahang, Pekan, Pahang, Malaysia \\ 7 WHO Collaborating Centre on eHealth, UNSW Digital Health, University of New South Wales, NSW 2052, Australia
}

Corresponding Authors: Md Nahidul Islam, Mohammad Ali Moni

Email address: nahidul76.edu@gmail.com, m.moni@unsw.edu.au

Hearing deficiency is the world's most common sensation of impairment and impedes human communication and learning. Early and precise hearing diagnosis using electroencephalogram (EEG) is referred to as the optimum strategy to deal with this issue. Among a wide range of EEG control signals, the most relevant modality for hearing loss diagnosis is auditory evoked potential (AEP) which is produced in the brain's cortex area through an auditory stimulus. This study aims to develop a robust intelligent auditory sensation system utilizing a pre-train deep learning framework by analyzing and evaluating the functional reliability of the hearing based on the AEP response. First, the raw AEP data is transformed into time-frequency images through the wavelet transformation. Then, lower-level functionality is eliminated using a pre-trained network. Here, an improved-VGG16 architecture has been designed based on removing some convolutional layers and adding new layers in the fully connected block. Subsequently, the higher levels of the neural network architecture are fine-tuned using the labelled timefrequency images. Finally, the proposed method's performance has been validated by a reputed publicly available AEP dataset, recorded from sixteen subjects when they have heard specific auditory stimuli in the left or right ear. The proposed method outperforms the state-of-art studies by improving the classification accuracy to $96.87 \%$ (from $57.375 \%$ ), which indicates that the proposed improved-VGG16 architecture can significantly deal with AEP response in early hearing loss diagnosis. 


\section{Diagnosis of Hearing Deficiency using EEG based}

2 AEP Signals: CWT and improved-VGG16 Pipeline

3

4

5

6

7

8
Md Nahidul Islam ${ }^{1}$, Norizam Sulaiman ${ }^{1}$, Fahmid Al Farid², Jia Uddin ${ }^{3}$, Salem A. Alyami ${ }^{4}$, Mamunur Rashid $^{1}$, Anwar P. P. Abdul Majeed ${ }^{5,6}$, Mohammad Ali Moni ${ }^{7}$

${ }^{1}$ Faculty of Electrical and Electronics Engineering Technology, Universiti Malaysia Pahang, Pekan, Pahang, 26600, Malaysia

${ }^{2}$ Faculty of Computing and Informatics, Multimedia University, Cyberjaya, 63000, MALAYSIA

${ }^{3}$ Technology Studies Department, Endicott College, Woosong University, Daejeon, South Korea

${ }^{4}$ Department of Mathematics and Statistics, Imam Mohammad Ibn Saud Islamic University, Saudi Arabia

${ }^{5}$ Innovative Manufacturing, Mechatronics and Sports Laboratory, Faculty of Manufacturing and Mechatronic Engineering Technology, Universiti Malaysia Pahang, Pekan, Pahang, Malaysia. ${ }^{6}$ Centre for Software Development \& Integrated Computing, Universiti Malaysia Pahang, Pekan, Pahang, Malaysia

${ }^{7} \mathrm{PhD}$, WHO Collaborating Centre on eHealth, UNSW Digital Health, University of New South Wales, NSW 2052, Australia

Corresponding Author:

Md Nahidul Islam ${ }^{1}$

Mohammad Ali Moni ${ }^{7}$

${ }^{1}$ Faculty of Electrical \& Electronics Engineering Technology, Universiti Malaysia Pahang, 26600 Pekan, Pahang, Malaysia

${ }^{7} \mathrm{PhD}$, WHO Collaborating Centre on eHealth, UNSW Digital Health, University of New South Wales, NSW 2052, Australia

E-mail address: nahidul76.edu@gmail.com, m.moni@unsw.edu.au 
39

40

41

42

43

44

45

46

47

48

49

50

51

52

53

54

55

56

57

58

59

60

61

62

63

64

65

66

67

68

69

70

71

72

73

74

75

76

77

78

\section{Abstract}

Hearing deficiency is the world's most common sensation of impairment and impedes human communication and learning. Early and precise hearing diagnosis using electroencephalogram (EEG) is referred to as the optimum strategy to deal with this issue. Among a wide range of EEG control signals, the most relevant modality for hearing loss diagnosis is auditory evoked potential (AEP) which is produced in the brain's cortex area through an auditory stimulus. This study aims to develop a robust intelligent auditory sensation system utilizing a pre-train deep learning framework by analyzing and evaluating the functional reliability of the hearing based on the AEP response. First, the raw AEP data is transformed into time-frequency images through the wavelet transformation. Then, lower-level functionality is eliminated using a pre-trained network. Here, an improved-VGG16 architecture has been designed based on removing some convolutional layers and adding new layers in the fully connected block. Subsequently, the higher levels of the neural network architecture are fine-tuned using the labelled time-frequency images. Finally, the proposed method's performance has been validated by a reputed publicly available AEP dataset, recorded from sixteen subjects when they have heard specific auditory stimuli in the left or right ear. The proposed method outperforms the state-of-art studies by improving the classification accuracy to $96.87 \%$ (from $57.375 \%$ ), which indicates that the proposed improved-VGG16 architecture can significantly deal with AEP response in early hearing loss diagnosis.

\section{Introduction}

Hearing deficiency is the widespread form of human sensory disability; it is the partial or complete inability to listen to the ear's sound. The world health organization (WHO) reports that 466 million people were living with hearing loss in 2018, projected to exceed 630 million by 2030 and more than 900 million by 2050 ("Deafness and hearing loss"). An early and effective hearing screening test is essential for address the vast population concern. That helps to reduce the hearing deficiency by taking necessary steps at an appropriate time. Conventional listening tests and audiograms appear to be subjective assessments that significantly demand medical and health services. The audiogram reflects the hearing threshold across the speech frequency spectrum, usually between 125 and $8000 \mathrm{~Hz}$. The traditional hearing impairment testing technique is very time-consuming, takes sufficient clinical time and expertise to interpret and maintain since it requires the person to respond directly. In the application of hearing aid, other issues, such as hearing loss's consequence (Holmes, Kitterick \& Summerfield, 2017), the circumstances of the auditory stimulus (such as the background noise of the stimulus, locations of the stimulus (Das, Bertrand \& Francart, 2018)(Das et al., 2016) ), attention-altering techniques is still an open question.

Various hearing impairment testing techniques have been conducted to address these issues, and among them, EEG-based auditory evoked potentials (AEPs) are most widely used (Zhang et al., 2006)(Mahmud et al., 2019). Nowadays, the classification of AEP signal is most commonly used in many brain-computer interface (BCI) applications (Gao, Wang \& Gao, 2014) and brain hearing issues (Sriraam, 2012). In fact, the AEP signal is widely used to recognize 
79 hearing capability, assessment, and neurological hearing impairment identification. The AEP 80 signals are reflected by the brain's electrical activity changes in the body's sensory mechanisms in 81 response to the auditory stimulus. The diagnosis of hearing loss typically involves four main 82 stages: acquisition of data, data pre-processing, feature extraction and selection, and classification. 83 The feature extraction is traditionally conducted by analyzing the time-domain, frequency-domain, 84 and time-frequency domain techniques, which help to extract the information from the original 85 raw data. The extracted features are then used as an input to the machine learning or deep learning 86 models for training. However, traditional diagnosis methods have some drawbacks. For example, 87 88 traditional hearing loss approaches are often based on manual feature selection. As a consequence, if the manually chosen features are ineffective for this task, the hearing loss recognition performance will decrease considerably. Furthermore, Handcrafted features for different classification tasks are task-specific, meaning that features that render predictions correctly are not acceptable under certain conditions for other scenarios (Acir, Erkan \& Bahtiyar, 2013)(Acir, Özdamar \& Güzeliş, 2006).

Although the researchers have employed a wide range of machine learning and deep learning algorithms, recognizing the most effective classifier is still an open question. Among machine learning-based classifiers, support vector machine (SVM) (Mahmud et al., 2019), k-nearest neighbors (k-NN) (Thorpe \& Dussard, 2018)(Rashid et al., 2021), artificial neural network (ANN) (Mccullagh et al., 1996), linear discriminant analysis (LDA) (Grent-'t-Jong et al., 2021) Naïve Bayesian (NB) (Shirzhiyan et al., 2019) are widely used in neurological response classification. Nowadays, the convolutional neural networks (CNNs) are the most preferred approach in the different classification tasks, particularly in image classification (Lecun, Bengio \& Hinton, 2015). In some recent studies, CNNs have shown promising performances in EEG signal classification: in seizure detection (Ansari et al., 2019), depression detection (Liu et al., 2018), and sleep stage classification (Ansari et al., 2018). Ciccarelli et al. (Ciccarelli et al., 2019) proposed a novel architecture of the neural network and showed that their approach outperforms the linear methods in decision windows of 10s. They have used eleven subjects in the experiment: with the wet EEG, the decoding accuracy was improved from $66 \%$ to $81 \%$, and with the dry EEG, the decoding accuracy was improved from 59\% to 87\%. McKearney et al. (McKearney \& MacKinnon, 2019) used a deep neural network approach to classify paired auditory brainstem responses. They used 232 paired ABR waveforms (190 paired ABR waveforms for training the model and 42 paired waveforms for performance evaluation) from eight normal hearing subjects and achieved 92.9\% testing accuracy. Although they achieved an excellent performance to identify the auditory brainstem response, the testing set is too small, and more dataset is needed to test the model performance. Mccullagh et al. (Mccullagh et al., 1996) reported a 73.7\% accuracy using the artificial neural network to classify 166 auditory brainstem responses (ABRs) with 2000 repetitions. Ibrahim et al. (Ibrahim, Ting \& Moghavvemi, 2019) used multiple classification techniques for detecting the hearing condition; the SVM algorithm outperforms the other 117 algorithms by achieving a classification accuracy of $90 \%$. They used a nonlinear feature extraction 118 method to extract adequate information from the AEP signals. Dietll et al. (Dietl \& Weiss, 2004) 
119 evaluated an application to achieve detection of frequency-specific hearing loss where they used 120 the wavelet packet transform (WPT) as a feature extraction method and support vector machines 121 (SVM) classifier to transient evoked otoacoustic emissions (TEOAE). They achieved a maximum 122 of $74.7 \%$ accuracy with the testing dataset. Nonetheless, the overall accuracy is not favourable 123 enough to be utilized in real-life applications. Tang et al. (Tang \& Lee, 2019) proposed a novel 124 hearing deficiency diagnosis method using three-level wavelet entropy, followed by MLP, trained 125 by hybrid Tabu search-Particle Swarm Optimization (TS-PSO). Their approach achieved 86.17\% 126 testing accuracy; it still needs improvement for real-time applications. Sanjay et al. (Sanjay et al., 127 2020) used machine learning approaches for human auditory threshold prediction. The absolute 128 threshold test (ATT) method was used for feature extraction from the auditory signals. The

129

130

131

132

133

134

135

136

137

138

139

140

141

142

143

144

145

146

147

148

149

150

151

152

153

154

155

156

157

158 extracted feature was then classified using multiple classification methods. Among all the classification methods, a maximum of $93.94 \%$ accuracy was achieved with the SVM classifier. Xue et al. (Xue et al., 2018) used participants' articulatory movements with or without hearing impairment during nasal finals for hearing impairment diagnosis. Six different kinematic features: standard deviation of velocity, minimum velocity, maximum velocity, mean velocity, duration, displacement was used to extract the information from the hearing impairment (HI) patient and normal hearing $(\mathrm{NH})$ participants. The classification was conducted with a support vector machine, radial basis function network, random forest, and $\mathrm{C} 4.5$. The maximum accuracy was $87.12 \%$ using a random forest classifier via (displacement and duration feature). Zhang et al. (Zhang et al., 2006) proposed an auditory brainstem response classification method. They used wavelet analysis for feature extraction and Bayesian networks to classify the auditory responses. Discrete wavelets transform (DWT) was used to extract the time-frequency information from the raw signals. A maximum of $78.80 \%$ testing accuracy was achieved in their proposed approach; it needs more improvement in testing accuracy.

The emphasis in our study is on a concise decision window. However, a concise window contains less information and more difficult to achieve high performance but provide an effective solution for early detection of hearing disorder. The short decision window is considered one of the prerequisites to develop the real-life application, but limited studies have been carried out to investigate this issue (Deckers et al., 2018). Moreover, selecting a short decision window makes the system faster by reducing the computational complexity of the system. On the other hand, Deep learning (DL) approaches can provide an effective solution because of their effective feature learning capability to overcome the above limitations (Krizhevsky, Sutskever \& Hinton, 2017)(Nossier et al., 2019)(Shao et al., 2019)(Bari et al., 2021)(Mahendra Kumar et al., 2021). Deep learning models have several hidden layers that can explicitly learn hierarchical representations. From model training, deep architectures can select discriminatory representations, which are helpful for precise predictions according to the training data in subsequent classification stages. Although the DL models have successful application in hearing loss diagnosis tasks, there are still some issues with DL approaches. A few investigations (Ciccarelli et al., 2019) (McKearney \& MacKinnon, 2019) have been conducted using deep models with more than ten hidden layers for hearing loss diagnosis. A large number of labelled data and computations 
159

160

161

162

163

164

165

166

167

168

169

170

171

172

173

174

175

176

177

178

179

180

181

182

183

184

185

186

187

188

189

190

191

192

193

194

195

196

197

resources are typically required during the training model from scratch. In the proposed study, we used the transfer learning (TL) method to address the challenges of training a deep model from scratch. The TL method is used to expedite the deep learning model training phase and effectively learns the hierarchical representations. The process is accomplished by using the pre-trained TL method that has been pre-trained on vast datasets of natural images. The proposed pre-trained model provides the lower-level weights for the target neural network, while the higher-level weights are fine-tuned for the hearing deficiency diagnosis task. Consequently, the proposed TL method offers a rational initialization for the target model and decreases the number of model's parameters. In this manner, TL significantly enhances the performance of the training process. Here, we summarized the main contribution of this paper.

- We have presented a hearing deficiency identification system based on deep CNN, where a transfer learning strategy has been used to improve the training process. To fit the AEP dataset in our model, we fine-tune the high-level parameters, consisting of unfreezing some part of the pre-training model and re-training it. The lower-level parameters are transferred from the previous trained deep architecture.

- In the proposed approach, we also changed some high-level parameters, reduced the number of parameters and complexity of the TL architecture, which helps in improving the performance of the VGG16 model for our dataset and reduces the computational time of the training process.

- The experiment is conducted in a short decision window (1s and $2 \mathrm{~s}$ ), minimizing the impact of additional features and reducing time consumption, which shows the proposed system robustness and applicability in real-life application.

The rest of the manuscript is arranged as follows: a detailed data description, data preprocessing, and the transformation process of CWT are implemented in the Materials and Methodology section. A detailed description of the development of the proposed pre-trained model and fine-tuning procedure for hearing deficiency diagnosis is also described in this section. Experimental performance to determine the models' validation is described in the Result of the Experiment and Analysis section. The Discussion section exhibits a discussion on the comparison of the proposed model with related studies, along with the key advantages of our proposed method over the previous studies. The Conclusion section represents the outcome of the present study.

\section{Materials and Methodology}

The aim of this study is to build an intelligent auditory sensation system for hearing loss diagnosis with high performance. The overall procedure of the proposed hearing loss diagnosis method is demonstrated in Figure 1. The proposed framework consists of few steps, including data collection, pre-processing, time-frequency analysis, and building a pre-trained model with fine-tuning. We have used a publicly available online dataset in the data collection phase instead of data collection ourselves. We converted the raw signal into a time-frequency image using continuous wavelet 
198

199

200

201

202

203

204

205

206

207

208

209

210

211

212

213

214

215

216

217

218

219

220

221

222

223

224

225

226

227

228

229

230

231

232

233

234

235

236

237

transform (CWT). Then, the proposed deep CNN (improved-VGG16) method is applied in the time-frequency images for diagnosis the hearing loss. In the TL model, the pre-trained ImageNet dataset has been used, and the size of the images is $224 * 224$ pixels in RGB. The entire dataset has been converted into a time-frequency image after data collection and resized in height-224* width-224* depth-3. The VGG16 uses natural images which are different from the time-frequency images of AEP. So, to fit the AEP dataset in the TL model, we replaced some VGG16 layers with the new layers and then fine-tuned the improved VGG16 model.

Figure 1: The overall procedure of hearing deficiency diagnosis method.

\section{Data Description}

Experimental AEP datasets are provided by ExpORL, Dept. Neurosciences, KULeuven, and Dept. Electrical Engineering (ESAT), KULeuven (Das, Francart \& Bertrand, 2020). A 64-channel BioSemi Active Two system was used for recording the AEP data, which was $8196 \mathrm{~Hz}$ sampling rate. The entire data was collected from 16 normal-hearing subjects, and the trial was repeated 20 times from each subject. The recordings were conducted in a soundproof, electromagnetically shielded space. The auditory stimuli were presented at $60 \mathrm{dBA}$ by Etymotic ER3 insert earphones and were low-pass filtered with a cut-off frequency of $4 \mathrm{kHz}$. As simulation software, APEX 3 was used (Francart, van Wieringen \& Wouters, 2008). Three male Flemish speakers narrated four Dutch stories as auditory stimulation ("Radio books for children"). Every story lasted 12 minutes and was divided into two segments of 6 minutes each. Silent segments that lasted more than 500 milliseconds were shortened to 500 milliseconds. The stimuli were equal in root-mean-square intensity and perceived as equally loud. The experiment was divided into eight sections, each lasting six minutes. Subjects were presented with two parts of two storylines in each trial. The left received one part, while the right ear received the other part. To prevent the lateralization bias described by (Das et al., 2016), the attended ear was alternated over successive trials to ensure that each ear received an equal volume of data. Each subject received stimuli in the same order, either dichotically or after head-related transfer function (HRTF) filtering (simulating sound coming from $\pm 90^{\circ}$ ). As with the attended ear, the HRTF/dichotic condition was randomized and balanced within and over subjects.

\section{Data Preprocessing}

The pre-processing of the AEP data is the first phase after data collection. In this study, the trials were filtered with a high pass ( $0.5 \mathrm{~Hz}$ cut off) and downsampled from the sampling rate of 8192 $\mathrm{Hz}$ to $128 \mathrm{~Hz}$. Here, we have investigated sixteen subjects, and each trial has been segmented into the same length. The entire dataset has been segmented into short decision windows (1s and $2 \mathrm{~s}$ ) and considered each decision window an observation. The straightforward reason to select the concise decision windows is to reduce the computational complexity and make the system faster, which will help detect the early hearing disorder. From each subject, 200 observations have been picked, and finally, we achieved a total of 3200 observations. After data filtering and window

Peer) Comput. Sci. reviewing PDF | (CS-2021:03:59631:1:0:NEW 6 Jun 2021) 
238

239

240

241

242

243

244

245

246

247

248

249

250

251

252

253

254

255

256

257

258

259

260

261

262

263

264

265

266

267

268

269

270

271

272

selection, the AEP data of subject-1, channel-1 in the time domain, is shown in Figure 2 when the subject hears auditory stimulus through headphones defined as left and right labels.

Figure 2: AEP raw data plotting in 2s decision window: (A) hear auditory stimulus with the left ear (B) hear auditory stimulus with the right ear.

\section{CWT for Time-Frequency Analysis}

CWT is a time-frequency feature extraction approach that offers multi-scale signal refinement by scaling and translating operations. After the data pre-processing step, the segmented dataset transforms from the time domain to the time-frequency domain using the CWT.

The CWT can automatically adapt the time-frequency signal analysis criteria and clearly explain the signal frequency change with time (Yan, Gao \& Chen, 2014). The CWT is widely used for feature extraction and can be considered a mathematical tool for transforming time-series into a different feature space. This study uses CWT as a feature extraction method that converts the raw signal into 2-D time-frequency images from 1-D time-domain signals. An internal signal operation and a series of wavelets are performed by the wavelet transforms. The mother wavelet is scaled and translated to create the wavelet set, which is a family of wavelets $\psi(t)$, shown as

$$
\psi_{S, \tau}(t)=\frac{1}{\sqrt{S}} \psi\left(\frac{t-\tau}{S}\right)
$$

Here, $S$ represents the scale parameter inversely related to frequency, and $\tau$ represents the translation parameter.

The signal $x(t)$ can be achieved by a complex conjugate convolution operation, mathematically defined as follows (Huang \& Wang, 2018):

$$
W(s, \tau)=\left\langle x(t), \psi_{S, \tau}\right\rangle=\frac{1}{\sqrt{s}} \int x(t) \psi^{*}\left(\frac{t-\tau}{S}\right) d t
$$

Where $\Psi^{*}(\cdot)$ denotes the complex conjugate of the above function $\Psi(\cdot)$ and This operation decomposes the signal $x(t)$ in a series of wavelet coefficients, in which the base function is the wavelet family. In the equation, the $s$ and $\tau$ are two types of parameters in the family wavelets. The signal $\mathrm{x}(\mathrm{t})$ is transformed and projected to the time and scale dimensions of the family wavelets.

In this study, we use wavelet basis functions (Mother Wavelets). The time-frequency images are then used as the input of the proposed TL model. The transformation process of CWT is shown in Figure 3.

Figure 3: The Transformation process from time-domain signal to time-frequency domain image. 
273

274

275

276

277

278

279

280

281

282

283

284

285

286

287

288

289

290

291

292

293

294

295

296

297

298

299

300

301

302

303

304

305

306

307

Finally, we concatenate the 64 channels data in $\left(\mathrm{M}^{*} \mathrm{M}\right)$ for preparing an observation, where the value of $M$ is set to 8 . So, each observation provides the time-frequency information of 64 channels. Figure 4 shows the time-frequency image of 64 channels.

Figure 4: The time-frequency image of 64 channels data.

\section{Hearing Deficiency Diagnosis using Deep TL.}

The proposed system presented a deep TL method based on improved-VGG16 architecture for hearing loss diagnosis. The VGG16 uses natural images which are different from the timefrequency images of AEP. The improvement consists of replacing some VGG16 layers with the new layers and then fine-tuning the layers to fit the time-frequency AEP dataset in the model.

\section{Convolutional Neural Network Architecture}

LeCun (LeCun et al., 1998) proposed the convolutional neural networks (CNN), one of the best pattern recognition methods. The locally trained filters are used in this system to extract the visual features through the input image. CNN's internal layer structure consists of a convolution layer, pooling layer, and fully connected layer. The complete procedure of CNN is shown in Figure. 5.

Figure 5: Typical Convolutional Neural Network Architecture.

\section{- Convolution Layer}

The convolutional operations provide the more advanced feature representation. Several fixed-size filters allow the complex functions to be used in the input image (Ravi et al., 2017). The same weights and bias values are used in the whole image in each filter. This technique is called the weight-sharing mechanism, and it makes it possible to represent the entire image with the same characteristic. A neuron's local receptive field reflects the neuron's region in the previous layer. This study uses the 'ReLU activation function (Nielsen). Let $\mathrm{c} \times \mathrm{c}$ is the size of the kernel or filter, and $i$ represent the time-frequency image. The weight and bias of the filter are denoted by w and b, respectively. The output $O_{0,0}$ can be computed using Eq. (3), where $f$ denotes the activation function. This study used the ReLU activation function. In most of the classification tasks, the ReLU activation function has demonstrated superior performance in terms of accelerating convergence and mitigating the issue of vanishing gradients (Krizhevsky, Sutskever \& Hinton, 2017). The mathematical representation of the ReLU activation function can also be seen in Eq. (4),

$$
\begin{gathered}
O_{0,0}=f\left(b+\sum_{t=0}^{c} \sum_{r=0}^{c} w_{t, r} i_{0+t, 0+r}\right) \\
f(x)=\left\{\begin{array}{ll}
x & x>0 \\
0 & \text { else }
\end{array} .\right.
\end{gathered}
$$


308

309

310

311

312

313

314

315

316

317

318

319

320

321

322

323

324

325

326

327

328

329

330

331

332

333

334

335

336

337

338

339

340

341

342

343

- Pooling Layer

The pooling method is used in the feature maps, which have gone through convolution and activation function. The pooling layer computes the local average or maximum value, reducing the complexity and retaining the essential features, thus enhancing feature extraction performance.

- Fully connected layer

The convolutional and pooling layers alternately transfer the image features; after that, the fully connected layer received the image feature as an input. One or more hidden layers may have in the fully connected layer. By the data from the previous layer, each neuron multiplies the connection weights and adds a bias value. Before transmission to the next layer, the measured value is passed via the activation function. Eq. (5). displays neuronal calculations in this layer.

$$
f c 1=f\left(b+\sum_{q=1}^{M} w_{1, q} * O_{q}\right)
$$

Where $\mathrm{f}$ is the activation function, $\mathrm{w}$ is the weight vector, $\mathrm{O}$ is the input vector of the $q^{\text {th }}$ neuron, and $b$ is the bias value.

\section{- SoftMax}

The SoftMax activation function variates the logistic regression adapted to multiple classes and used in the output layer for classification purposes. It can be determined by Eq. (6) (Sermanet et al., 2013),

$$
\operatorname{class}_{j}=\frac{\exp \left(s f_{j}\right)}{\sum_{q} \exp \left(s f_{q}\right)}
$$

\section{Proposed Pretrained Model Building and Fine-tuning}

In the convolutional neural network, the convolutional layers are used to extract the features from the dataset in a different manner, whereas the fully connected layers are used to classify the extracted features. The most forthright approach for enhancing the feature learning capability is to increase the depth or width of the deep neural network. However, this can lead to two issues: the first concern is that a deeper or wider model typically has more parameters, rendering the expanded network more vulnerable to overfitting. The second concern is that it raises the use of computing resources substantially.

To overcome these flowing issues and extract the AEP feature efficiently, the VGG16 network utilizes several parallel layers with different convolutional kernel sizes. It concatenates the outputs at the end of the pre-trained network. In the proposed TL model, we replace some layers of VGG16 with the new layers to fit the AEP dataset in the pre-trained network, which enhances hearing loss identification performance. The replacement process consists of adding 
344

345

346

347

348

349

350

351

352

353

354

355

356

357

358

359

360

361

362

363

364

365

366

367

368

369

370

371

372

373

374

375

376

377

378

379

380

381

382

383

some dense layers in the fully connected block of VGG16 architecture and adding the dropout layers after every dense layer. A densely connect layer learns features from all the previous layer's features. The dense layer performs a matrix-vector multiplication, and with the help of backpropagation, the parameters can be trained and updated. The dense layer is used to change the vector's dimensions and applies in other operations like rotation, scaling, and translation. Mele \& Altarelli (Mele \& Altarelli, 1993) reported that on the CIFAT-10 dataset, the error rate 16.6\% when testing the dataset in a convolutional neural network. They improved the model's performance with an error rate of $15.6 \%$ when the dropout layer was utilized in the last hidden layer. We add the dropout layer after every dense layer in the fully connected block to reduce the model complexity and prevent overfitting. The neuron is temporarily dropped with the probability $\mathrm{p}$ at each iteration. Then, at every training step, the dropped-out neuron is resampled with the probability $p$, and a dropped-out neuron will be active at the next step. Here, the hyperparameter $p$ is the dropout rate. Since the VGG16 uses the 'ImageNet' weight, which is trained with the natural image, and the proposed time-frequency images are not similar, more layers need to be fine-tuned where the weight is updated with the 'ImageNet' weight. This process helps to fit the timefrequency images with the TL architecture. The proposed fine-tuning consists of unfreezing some pre-trained network layers and re-train with the AEP dataset.

In the proposed approach, at first, we remove all the layers of VGG16 after the first $3 \times 3$ convolution layer of convolutional block 5, as shown in Figure 6, and replace the fully connected block there. Then, we add multiple dense layers at the end of the VGG16 model, and after every dense layer, we add the dropout layer. In the case of $\mathrm{CNN}$, the convolutional layers extract the feature from the dataset, whereas the fully connected layers try to classify the extracted features. Consequently, adding more layers to the dense section can empower the network's robustness and improve classification accuracy. So, despite using the two dense layers of the VGG16, here, we add three new dense layers units of 1024, 512, and 288 in the fully connected block. Then, we add a dropout layer after each dense layer, and the dropout value is set to $0.2,0.4$, and 0.6 , respectively. The reason behind adding the dropout layers is that the deep learning model reduces the performance due to overfitting, and the dropout layers reduce the model complexity and prevent overfitting. These techniques help in enhancing the performance in the hearing loss diagnosis. We also remove the top layer and adding a SoftMax layer (output layer) based on the targeted class. Based on the hyperparameters tuning technique, the proposed approach uses the 'Adam' optimizer to adjust the network weight with the batch size 64, and the learning rate is set to 0.0001 . The parameters selection is made with the help of the 'Keras-Tuner' library. This library helps to select the most optimal set of hyperparameters for our architecture. Hyperparameters are the variable that governs the training process of the DL model and structure. There are two types of hyperparameters: first, model hyperparameters that help in selecting the number and width of the multiple hidden layers. Second, algorithm hyperparameters help to influence the speed and quality of the learning algorithm. All the hyperparameters selected to build the proposed architecture are based on ten different runs of the model. The following steps are used to train the model for hearing loss identification, shown in Box 1.

Peer) Comput. Sci. reviewing PDF | (CS-2021:03:59631:1:0:NEW 6 Jun 2021) 
384

385

386

387

388

389

390

391

392

393

394

395

396

397

398

399

400

401

402

403

404

405

406

407

408

409

410

411

412

413

414

415

416

417

418

419

420

421

422

423

Box 1: Training procedure of proposed TL architecture.

The detailed information of the parameter of the proposed TL architecture is shown in Table 1. Here, $\mathrm{C}$ means the targeted class.

Table 1: Parameter of proposed TL architecture.

During the training process, all the layers before convolutional block four are frozen. The weights are updated in the trainable layers, which helps in minimizing the errors between the predicted labels and the actual labels. The complete architecture of the proposed TL has demonstrated in Figure 6.

Figure 6: Transfer Learning Procedure of the Proposed Method.

\section{Result of the Experiment and Analysis}

This section represents the proposed hearing loss diagnosis method's performance based on CWT and deep CNN architecture (improved-VGG16). First, we converted the time domain signal to time-frequency domain images. Then, the images are resized into height- $224 *$ width-224* depth3 , which is the suitable size of the proposed model. In this study, two different decision windows were tested: $1 \mathrm{~s}$ and $2 \mathrm{~s}$. This term refers to the quantity of data required to make a single left/right decision. The practical reason behind selecting the shorter decision window is to detect the hearing condition quickly. The entire dataset was randomly split into the training set and testing set. Here, we used $70 \%$ dataset to train the architecture, and the rest of the dataset was used to test the model's validation. This experiment has conducted with sixteen subjects where the subjects hear the auditory track. Based on listening to the auditory track with the ear, the dataset has been divided into two classes. The 'Class1' means the subject hears the auditory track with the left ear and the 'Class2' means the subject hears the auditory track with the right ear. With the (1s and 2s) decision windows, we randomly selected 200 observations from each subject. A total of 2240 observations has been used for training the model and 960 observations for testing the performance.

For 1s window length, the performance of the proposed approach for each subject in terms of accuracy, precision, recall, f1-score and cohen's kappa of all subjects is demonstrated in Table 2.

Table 2: Performance of proposed model for 1s decision window.

Table 2 illustrates that in the case of subject-5, subject-7, and subject-16, our network achieves an unprecedented performance of 100\%. Except for six subjects (Subjects-3, 6, 9, 11, 13 and 14), all subjects have achieved more than 90\% accuracy. However, comparatively lower classification accuracy has been noticed by Subjects-3 (86.67\%), Subject-6 (83.33\%), Subject-9 (76.67\%), 
424

425

426

427

428

429

430

431

432

433

434

435

436

437

438

439

440

441

442

443

444

445

446

447

448

449

450

451

452

453

454

455

456

457

458

459

460

461

462

463

Subject-11 (81.67\%), Subject-13 (78.33\%), and Subject-14 (76.67\%). In the case of 1s decision window length, the average classification accuracy is $91.56 \%$, whereas the standard deviation is 8.91\%. Besides classification accuracy, other performance evaluation techniques (such as precision, recall, fl-score, and cohen kappa score) are also calculated to check the proposed model's acuity. The average value of precision, recall, fl-score, and cohen kappa for sixteen subjects are $90.74 \%, 93.63 \%, 91.92 \%, 82.71 \%$, respectively, whereas standard deviations are $10.47 \%, 8.25 \%, 8.79 \%, 18.34 \%$, respectively. Figure 7 shows the overall accuracy and loss curve of the proposed TL method for the $1 \mathrm{~s}$ decision window.

Figure 7: The overall accuracy and loss curve of the proposed TL method for 1s decision window.

For $2 \mathrm{~s}$ window length, the performance of the proposed architecture is illustrated in Table 3. In this case, a maximum of $100 \%$ accuracy has achieved for subject-6, subject-7, subject-10, subject-16. Here, in the case of subject-16, we achieved $1.67 \%$ more accuracy compared to the $1 \mathrm{~s}$ time window analysis. However, the proposed architecture achieves an unprecedented improvement (more than or equal to $90 \%$ for decision windows of $2 \mathrm{~s}$ ) in each subject. The lowest accuracy of $90 \%$ has been obtained in subject- 13 .

Table 3: Performance of proposed model for 2 s decision window.

With the $2 \mathrm{~s}$ decision window, the average value of accuracy precision, recall, fl-score, and cohen kappa for sixteen subjects are $96.87 \%, 96.49 \%, 97.57 \%, 97 \%$ and $93.73 \%$, respectively. On the other hand, the standard deviation of precision, recall, f1-score, and cohen kappa are 2.78\%, $3.50 \%, 2.76 \%, 2.64 \%$ and $5.57 \%$, respectively. Figure 8 shows the overall accuracy and loss curve of the proposed TL method.

Figure 8: The overall accuracy and loss curve of the proposed TL method for $2 \mathrm{~s}$ decision window.

To illustrate the performance of the proposed TL model in depth, the confusion matrix of all subjects has been given separately. A confusion matrix can be used to estimate the classification accuracy of a model visually. Figure 9 represent the confusion matrix with $1 \mathrm{~s}$ decision windows analysis, whereas Figure 10 represent the confusion matrix with 2 s decision window analysis. In both figures, the letter $\mathrm{A}$ to $\mathrm{P}$ denotes the confusion matrix of subject-1 to subject-16, respectively.

Figure 9: Confusion matrix for 1s decision windows (A)subject1 (B)subject2 (C)subject3 (D)subject4 (E)subject5 (F)subject6 (G)subject7 (H)subject8 (I)subject9 (J)subject10 (K)subject11 (L)subject12 (M)subject13 (N)subject14 (O)subject15 (P)subject16

Peer] Comput. Sci. reviewing PDF | (CS-2021:03:59631:1:0:NEW 6 Jun 2021) 
464

465

466

467

468

469

470

471

472

473

474

475

476

477

478

479

480

481

482

483

484

485

486

487

488

489

490

491

492

493

494

495

496

497

498

499

500

501

502

503

504

Figure 10: Confusion matrix for 2s decision windows (A)subject1 (B)subject2 (C)subject3

(D)subject4 (E)subject5 (F)subject6 (G)subject7 (H)subject8 (I)subject9 (J)subject10

(K)subject11 (L)subject12 (M)subject13 (N)subject14 (O)subject15 (P)subject16

The correct predictions are on the diagonal in the confusion matrix, while the incorrect predictions are off the diagonal. For example, in the case of Figure 10(A) that denotes subject-1, a total of 59 observations (29 observations for class1, 30 observations for class2) have been recognized accurately among 60 observations. In both decision windows, the total testing set for sixteen subjects consists of 960 observations, in which 464 observations are in 'Class1', and 496 observations are in 'Class2'. For 1s decision windows, our network correctly detects 876 observations whilst 84 observations have been misclassified (shown in Figure 9). On the other hand, for 2 s decision windows, 930 observations have been accurately detected, whereas only 30 observations have been misclassified (shown in Figure 10). Therefore, $2 \mathrm{~s}$ decision windows provide a significant performance compared to the $1 \mathrm{~s}$ decision windows.

Furthermore, to study the relationship between window length and detection performance, this study includes a comparison. Figure 11 visualizes the average performance of two decision windows over our network.

Figure 11: Hearing deficiency detection performance of the proposed TL architecture for two different window lengths.

Figure 11 shows that the proposed TL network with a 2s decision window significantly improves recognition accuracy compared to the $1 \mathrm{~s}$ decision window analysis. The main goal of this study is to enhance the performance for detecting the hearing condition with a concise decision window, so that we can efficiently use this system in real-life application. For this purpose, first, we analyze the $1 \mathrm{~s}$ decision window and achieve $91.56 \%$ recognition accuracy; still not so high to apply this system in real-life application. Furthermore, to enhance the performance of our proposed diagnosis system, we move on to the $2 \mathrm{~s}$ decision windows length, and this time we achieve a $5.31 \%$ improvement in accuracy compared to the $1 \mathrm{~s}$ decision window length. In the case of other performance evaluation techniques such as precision, recall, F1 score and Cohen's kappa, we achieve $5.74 \%, 3.94 \%, 5.08 \%$, and $11.02 \%$, improvement, respectively. The improvement indicates the robustness and applicability of our proposed system.

Despite the impressive performance of the proposed system, in some cases, the performance of our network is unsatisfactory. The possible reason for this poorer performance compared to the other successful cases is that in EEG-based BCI application studies, a small SNR and different noise sources are among the greatest challenges. Furthermore, Unwanted signals contained in the main signal can be termed noise, artifact, or interference. Sometimes, the brain may produce some unwanted noise due to the lack of the subject's proper attention or muscle movement, affecting the detection results. In the experiment, we select concise decision windows (1s and $2 \mathrm{~s}$ ), and working with a short window have many advantages but still very challenging 
505 (Geirnaert, Francart \& Bertrand, 2020). For these possible reasons, some subjects may provide a

506

507

508

509

510

511

512

513

514

515

516

517

518

519

520

521

522

523

524

525

526

527

528

529

530

531

532

533

534

535

536

537

538

539

540

541

542

543

544

lower accuracy compared to the other's subject (shown in Table2, and Table 3). Suppose in the $2 \mathrm{~s}$ decision windows length; if we avoid the two subjects that perform poorer than the other subjects (shown in Table 3), we will achieve $97.62 \%$ recognition accuracy. However, the average training and testing accuracy of sixteen subjects with 2 s windows length is $100 \%$ and $96.87 \%$, respectively, after 100 epochs, whereas the standard deviation is $2.78 \%$.

Furthermore, to study the robustness of the proposed method with a $2 \mathrm{~s}$ decision window (1s decision windows is not considered in the subsequent analysis), the performance of the proposed model has been compared with other widely used TL architectures. Six popular transfer learning algorithms namely, InceptionResNetV2 (Längkvist, Karlsson \& Loutfi, 2014), MobileNet (Pan et al., 2020), ResNet50 (He et al., 2016), VGG16 (Simonyan \& Zisserman, 2015), VGG19 (Simonyan \& Zisserman, 2015), and Xception (Chollet, 2017) have employed to the timefrequency image of AEP dataset for hearing loss diagnosis. The input size is the same (height$224 *$ width-224* depth-3) for all the TL architectures. Figure 12 illustrates the performance comparison of six popular TL models with the proposed model. According to Figure 12, the proposed model achieved higher accuracy compared to the other TL models.

Figure 12: The performance comparison with other pre-trained architectures.

We also reduced the model parameters of VGG16 which help in reducing the model complexity and minimize the computational resources. The total number of all model parameters and performance is represented in Table 4 . Table 4 reported that the overall accuracy is less than $61 \%$ in all the pre-trained networks, where the models used pre-trained 'ImageNet' weights for hearing impairment identification.

Table 4: Performance comparison with six popular TL models.

In the proposed TL methods (Improved-VGG16), we reduced the total number of parameters of VGG16 $(134,268,738$ to $113,429,666)$. Although we reduced the number of parameters, the testing accuracy was still improved to $96.87 \%$ from $57.37 \%$. The reason behind the higher accuracy of the proposed model compared to the other TL models is the replacement of some VGG16's layers with the new layers and fine-tune the higher higher-level parameters, which helps to fit the AEP dataset in the pre-trained network. This replacement consists of adding some dense layers in the fully connected block of VGG16 architecture and adding the dropout layers after every dense layer (shown in Figure 6). In the fine-tuning block, the time-frequency images are updated with the 'ImageNet' weight. This technique helps to fit the dataset in the proposed TL architecture and enhance the overall performance for the hearing loss diagnosis. This experiment is carried out in python, where we used Google colab, Windows 10, Intel(R) Xeon(R) CPU @ 2.30GHz, Tesla K80, and CUDA Version: 10.1.

Peer] Comput. Sci. reviewing PDF | (CS-2021:03:59631:1:0:NEW 6 Jun 2021) 


\section{Discussion}

546 A hearing deficiency detection method based on CWT and improved-VGG16 is proposed in this 547 paper and achieved significantly outperform performance with the shorter decision windows (2s) 548 than the previous state-of-art studies. The proposed improved-VGG16 architecture achieved an 549 average accuracy, precision, recall, fl-score, and Cohen kappa of 96.87\%, 96.50\%, 97.58\%, $55097.01 \%$, and $93.74 \%$, respectively.

$551 \quad$ From Figure 12, it is clear that our network achieved more than 35\% significant 552 improvement compared to the others TL algorithms. In this experiment, we also found a significant 553 effect of the decision window length on the overall performance. We achieved the improvement 554 in the 2 s decision window: $5.31 \%$ accuracy, 5.74\% precision, 3.94\% recall, 5.08\% in F1 score, 555 and $11.02 \%$ Cohen's kappa than the 1s decision window. The improvement is because the concise

556

557

558

559

560

561

562

563

564

565

566

567

568

569

570

571

572

573

574

575

576

577

578

579

580

581

582

583

584 decision windows (1s) contain less information and sometimes provide unsatisfactory performance. However, this study aims to build an efficient network that can detect the hearing condition with a concise decision window so that we can able to achieve the decision quickly and can provide more effectiveness in real-life application.

Furthermore, a comparison of the proposed model with existing related studies is represented in Table 5. As seen in Table 5, Hallac et al. (Hallac et al., 2019) and Dass et al. (Dass, Holi \& Soundararajan, 2016) utilized the convolutional neural network-based classification approach and achieved higher accuracy compared to the other related studies. Hallac et al. (Hallac et al., 2019) reported that with the raw AEP data and CNN, they achieved $94.1 \%$ accuracy. Dass et al. (Dass, Holi \& Soundararajan, 2016) used both the time and frequency domain feature to extract the information from the raw AEP data. They used a feed-forward multilayer network to classify the AEP signal and achieved $90.74 \%$ testing accuracy. Both studies achieved a very encouraging performance but need more testing observations to validate the model's robustness.

Table 5: Performance comparison of related AEP studies.

In (Dietl \& Weiss, 2004)(Mahmud et al., 2019) (Tan et al., 2013) (Li et al., 2019), the SVM classifier was used to classify the AEP dataset. Their approach achieved $78.80 \%, 85.71,87 \%$, and $78.7 \%$ accuracy, respectively. The obtained overall performance is not enough to apply the models in real-life application. Tang et al. (Tang \& Lee, 2019) proposed a TS-PSO hybrid model to classify the two-class AEP dataset. They used Wavelet entropy as a feature extraction method and achieved $86.17 \%$ testing accuracy. Zhang et al. (Zhang et al., 2006) proposed a combination of wavelet analysis and Bayesian networks to classify auditory brainstem response (ABR) signals. For the wavelet analysis, they used the DWT method. Although they conducted an excellent analysis, the overall accuracy is reported $78.80 \%$, which needs improvement.

The experimental outcomes demonstrated that the proposed architecture gain an impressive performance than the other related study for hearing deficiency diagnosis reported in the literature. Although the proposed approach outperforms state-of-art hearing deficiency detection methods, some difficulties are also faced during the experimental analysis. For example, to check the cross- 
585

586

587

588

589

590

591

592

593

594

595

596

597

598

599

600

601

602

603

604

605

606

607

608

609

610

611

612

613

614

615

616

617

618

619

620

621

622

623

624

625

validation and prove the feasibility of our proposed network, a wide range of similar datasets is needed. However, we did not find such dataset for further validation of the proposed method. Another issue is the absence of clear speech envelopes in the dataset. In the previous research, several types of EEG headsets were used to detect the hearing conditions, and these contain a different number of electrodes (1-256). So, the number of electrodes and which electrodes are required to achieve acceptable performance should be determined (Mirkovic et al., 2015)(Montoya-Martínez, Bertrand \& Francart, 2019)(Narayanan \& Bertrand, 2018). In most of the studies, the analysis is carried out with ordinary machine learning algorithms, and a few studies are investigated with the deep learning approaches (Krizhevsky, Sutskever \& Hinton, 2017)(Nossier et al., 2019)(Shao et al., 2019). However, most of the studies' testing accuracy is not enough to use the model in real-time as well as real-life applications. A fast and more accurate approach can be an efficient tool for future hearing devices and provide a great application in reallife uses. Our study proposed the time-frequency distribution with a deep learning method and achieved superior performance to other related approaches for hearing loss diagnosis reported in the literature. The key advantages of our proposed method compared to previous studies are written below:

- Instead of training the AEP dataset with the deep learning architecture from scratch, the proposed study is conducted with a transfer learning strategy, which helps in faster training and better accuracy.

- To fit our time-frequency AEP dataset with the pre-trained model weight, we fine-tuned some higher-level parameters where the pre-trained weights are updated with the provided dataset. This strategy helps in enhancing the overall performance for detecting hearing deficiency.

- We compare the model's performance with the six popular TL methods, including VGG16 (Simonyan \& Zisserman, 2015), VGG19 (Simonyan \& Zisserman, 2015), MobileNet (Pan et al., 2020), ResNet50 (He et al., 2016), InceptionResNetV2 (Längkvist, Karlsson \& Loutfi, 2014), and Xception (Chollet, 2017) algorithms where the proposed architecture is superior for hearing deficiency diagnosis.

- We also changed some higher-level parameters (after the first layer of the convolutional block 5, we remove all the layers and add the new fully connected layer shown in Figure 5). This approach also helps in reducing the VGG16 parameters and increasing the performance of the proposed improved-VGG16 model.

- The proposed approach achieved the height classification accuracy of $96.87 \%$, compared to the previous studies (Ciccarelli et al., 2019) (McKearney \& MacKinnon, 2019) (Ibrahim, Ting \& Moghavvemi, 2019) (Dietl \& Weiss, 2004) (Tang \& Lee, 2019) (Sanjay et al., 2020) (Xue et al., 2018) (Zhang et al., 2006) (Tang \& Lee, 2019) (Mahmud et al., 2019) 
626

627

628

629

630

631

632

633

634

635

636

637

638

639

640

641

642

643

644

645

646

647

648

649

650

651

652

653

654

655

656

657

658

659

660

661

662

663

664

665

666

667

668

(Dietl \& Weiss, 2004) (Zhang et al., 2006) (Tan et al., 2013) (Li et al., 2019) (Hallac et al., 2019) (Dass, Holi \& Soundararajan, 2016).

- The impact of different decision windows is also exhibited in the proposed study, whereas our network provides a significant outcome with a concise decision window.

\section{Conclusions}

The proposed hearing loss diagnosis framework consists of two major steps: signal to image transformation and building a hearing deficiency diagnosis system using deep CNN. In the proposed study, the CWT is used to convert the raw signals to time-frequency images. Then, CNNbased improved-VGG16 is used to classify the time-frequency images. This approach achieved better outcomes with fewer trainable parameters, which help to reduce the training time of the model. The applicability and effectiveness of the proposed method are verified by the publicly available AEP dataset, and it achieved $96.87 \%$ testing accuracy with a concise decision window. Moreover, this study will help to identify early hearing disorders efficiently. Because of the unstable and subject-specific characteristics of the AEP signal, identification of the AEP signal is challenging. Thus, to enhance the detection system's accuracy, other AEPs features need to be investigated, and the use of more data variance and conditions can also be improved the outcome.

\section{Acknowledgements}

The authors would like to thank the Ministry of Higher Education for providing financial support under Fundamental research grant No. FRGS/1/2018/TK04/UMP/02/3 (University reference RDU190109) and Universiti Malaysia Pahang for laboratory facilities as well as additional financial support under Internal Research grant RDU190109.

\section{References}

Acir N, Erkan Y, Bahtiyar YA. 2013. Auditory brainstem response classification for threshold detection using estimated evoked potential data: Comparison with ensemble averaged data. Neural Computing and Applications 22:859-867. DOI: 10.1007/s00521-011-0776-2.

Acir N, Özdamar Ö, Güzeliş C. 2006. Automatic classification of auditory brainstem responses using SVM-based feature selection algorithm for threshold detection. Engineering Applications of Artificial Intelligence 19:209-218. DOI: 10.1016/j.engappai.2005.08.004.

Ansari AH, Cherian PJ, Caicedo A, Naulaers G, De Vos M, Van Huffel S. 2019. Neonatal Seizure Detection Using Deep Convolutional Neural Networks. International Journal of Neural Systems 29. DOI: 10.1142/S0129065718500119.

Ansari AH, De Wel O, Lavanga M, Caicedo A, Dereymaeker A, Jansen K, Vervisch J, De Vos M, Naulaers G, Van Huffel S. 2018. Quiet sleep detection in preterm infants using deep convolutional neural networks. Journal of Neural Engineering 15. DOI: 10.1088/17412552/aadc1f.

Bari BS, Islam N, Rashid M, Hasan J, Azraai M, Razman M, Musa RM. 2021. A real-time approach of diagnosing rice leaf disease using deep learning-based faster R-CNN framework. :1-27. DOI: 10.7717/peerj-cs.432.

Chollet F. 2017. Xception: Deep learning with depthwise separable convolutions. In: Proceedings

Peer] Comput. Sci. reviewing PDF | (CS-2021:03:59631:1:0:NEW 6 Jun 2021) 
669

670

671

672

673

674

675

676

677

678

679

680

681

682

683

684

685

686

687

688

689

690

691

692

693

694

695

696

697

698

699

700

701

702

703

704

705

706

707

708

709

710

711

712

713

714
- 30th IEEE Conference on Computer Vision and Pattern Recognition, CVPR 2017. Institute of Electrical and Electronics Engineers Inc., 1800-1807. DOI: 10.1109/CVPR.2017.195.

Ciccarelli G, Nolan M, Perricone J, Calamia PT, Haro S, O’Sullivan J, Mesgarani N, Quatieri TF, Smalt CJ. 2019. Comparison of Two-Talker Attention Decoding from EEG with Nonlinear Neural Networks and Linear Methods. Scientific Reports 9:1-10. DOI: 10.1038/s41598-01947795-0.

Das N, Bertrand A, Francart T. 2018. EEG-based auditory attention detection: Boundary conditions for background noise and speaker positions. Journal of Neural Engineering 15. DOI: 10.1088/1741-2552/aae0a6.

Das N, Biesmans W, Bertrand A, Francart T. 2016. The effect of head-related filtering and earspecific decoding bias on auditory attention detection. Journal of Neural Engineering 13. DOI: $10.1088 / 1741-2560 / 13 / 5 / 056014$.

Das N, Francart T, Bertrand A. 2020. Auditory Attention Detection Dataset KULeuven. DOI: 10.5281/ZENODO.3997352.

Dass S, Holi MS, Soundararajan K. 2016. Classification of brainstem auditory evoked potentials using artificial neural network based on time and frequency domain features. Journal of Clinical Engineering 41:72-82. DOI: 10.1097/JCE.0000000000000148.

Deafness and hearing loss. Available at https://www.who.int/news-room/factsheets/detail/deafness-and-hearing-loss (accessed June 18, 2020).

Deckers L, Das N, Ansari AH, Bertrand A, Francart T. 2018. EEG-based detection of the attended speaker and the locus of auditory attention with convolutional neural networks. bioRxiv:475673. DOI: 10.1101/475673.

Dietl H, Weiss S. 2004. Detection of cochlear hearing loss applying wavelet packets and support vector machines. In: Conference Record - Asilomar Conference on Signals, Systems and Computers. 1575-1579. DOI: 10.1109/acssc.2004.1399421.

Francart T, van Wieringen A, Wouters J. 2008. APEX 3: a multi-purpose test platform for auditory psychophysical experiments. Journal of Neuroscience Methods 172:283-293. DOI: 10.1016/j.jneumeth.2008.04.020.

Gao S, Wang Y, Gao X. 2014. Visual and Auditory Brain - Computer Interfaces. 61:1436-1447.

Geirnaert S, Francart T, Bertrand A. 2020. An Interpretable Performance Metric for Auditory Attention Decoding Algorithms in a Context of Neuro-Steered Gain Control. IEEE Transactions on Neural Systems and Rehabilitation Engineering 28:307-317. DOI: 10.1109/TNSRE.2019.2952724.

Grent-'t-Jong T, Gajwani R, Gross J, Gumley AI, Krishnadas R, Lawrie SM, Schwannauer M, Schultze-Lutter F, Uhlhaas PJ. 2021. 40-Hz Auditory Steady-State Responses Characterize Circuit Dysfunctions and Predict Clinical Outcomes in Clinical-High-Risk Participants: A MEG Study. Biological Psychiatry. DOI: 10.1016/j.biopsych.2021.03.018.

Hallac RR, Lee J, Pressler M, Seaward JR, Kane AA. 2019. Identifying Ear Abnormality from 2D Photographs Using Convolutional Neural Networks. Scientific Reports 9:1-6. DOI: 10.1038/s41598-019-54779-7.

He K, Zhang X, Ren S, Sun J. 2016. Deep residual learning for image recognition. In: Proceedings of the IEEE Computer Society Conference on Computer Vision and Pattern Recognition. IEEE Computer Society, 770-778. DOI: 10.1109/CVPR.2016.90.

Holmes E, Kitterick PT, Summerfield AQ. 2017. Peripheral hearing loss reduces the ability of children to direct selective attention during multi-talker listening. Hearing Research 350:160-172. DOI: 10.1016/j.heares.2017.05.005. 
715 Huang L, Wang J. 2018. Forecasting energy fluctuation model by wavelet decomposition and

716

717

718

719

720

721

722

723

724

725

726

727

728

729

730

731

732

733

734

735

736

737

738

739

740

741

742

743

744

745

746

747

748

749

750

751

752

753

754

755

756

757

758

759

760 stochastic recurrent wavelet neural network. Neurocomputing 309:70-82. DOI: 10.1016/j.neucom.2018.04.071.

Ibrahim IA, Ting HN, Moghavvemi M. 2019. Formulation of a Novel Classification Indices for Classification of Human Hearing Abilities According to Cortical Auditory Event Potential signals. Arabian Journal for Science and Engineering 44:7133-7147. DOI: 10.1007/s13369019-03835-5.

Krizhevsky A, Sutskever I, Hinton GE. 2017. ImageNet classification with deep convolutional neural networks. Communications of the ACM 60:84-90. DOI: 10.1145/3065386.

Längkvist M, Karlsson L, Loutfi A. 2014. Inception-v4, Inception-ResNet and the Impact of Residual Connections on Learning. Pattern Recognition Letters 42:11-24.

Lecun Y, Bengio Y, Hinton G. 2015. Deep learning. Nature 521:436-444. DOI: 10.1038/nature14539.

LeCun Y, Bottou L, Bengio Y, Haffner P. 1998. Gradient-based learning applied to document recognition. Proceedings of the IEEE 86:2278-2323. DOI: 10.1109/5.726791.

Li PZ, Huang L, Wang CD, Li C, Lai JH. 2019. Brain network analysis for auditory disease: A twofold study. Neurocomputing 347:230-239. DOI: 10.1016/j.neucom.2019.04.013.

Liu N, Lu Z, Xu B, Liao Q. 2018. Learning a convolutional neural network for sleep stage classification. In: Proceedings - 2017 10th International Congress on Image and Signal Processing, BioMedical Engineering and Informatics, CISP-BMEI 2017. Institute of Electrical and Electronics Engineers Inc., 1-6. DOI: 10.1109/CISP-BMEI.2017.8302226.

Mahendra Kumar JL, Rashid M, Musa RM, Mohd Razman MA, Sulaiman N, Jailani R, Abdul Majeed APP. 2021. The classification of EEG-based winking signals: A transfer learning and random forest pipeline. PeerJ 9. DOI: 10.7717/peerj.11182.

Mahmud MS, Yeasin M, Shen D, Arnott SR, Alain C, Bidelman GM. 2019. What brain connectivity patterns from EEG tell us about hearing loss: A graph theoretic approach. In: ICECE 2018 - 10th International Conference on Electrical and Computer Engineering. Institute of Electrical and Electronics Engineers Inc., 205-208. DOI: 10.1109/ICECE.2018.8636698.

Mccullagh PJ, King G, Mcallister HG, Houston HG. 1996. Classification of brainstem auditory evoked potentials using artificial neural networks. Studies in Health Technology and Informatics 34:547-550. DOI: 10.3233/978-1-60750-878-6-547.

McKearney RM, MacKinnon RC. 2019. Objective auditory brainstem response classification using machine learning. International Journal of Audiology 58:224-230. DOI: 10.1080/14992027.2018.1551633.

Mele B, Altarelli G. 1993. Dropout: A Simple Way to Prevent Neural Networks from Overfittin. Physics Letters B 299:345-350. DOI: 10.1016/0370-2693(93)90272-J.

Mirkovic B, Debener S, Jaeger M, De Vos M. 2015. Decoding the attended speech stream with multi-channel EEG: Implications for online, daily-life applications. Journal of Neural Engineering 12. DOI: 10.1088/1741-2560/12/4/046007.

Montoya-Martínez J, Bertrand A, Francart T. 2019. Optimal number and placement of EEG electrodes for measurement of neural tracking of speech Dynamical Systems, Signal Processing and Data Analytics Oto-Rhino-Laryngology Optimal number and placement of EEG electrodes for measurement of neural tracking of speech 2. bioRxiv:800979. DOI: $10.1101 / 800979$.

Narayanan AM, Bertrand AA. 2018. The effect of miniaturization and galvanic separation of EEG

Peer] Comput. Sci. reviewing PDF | (CS-2021:03:59631:1:0:NEW 6 Jun 2021) 
761

762

763

764

765

766

767

768

769

770

771

772

773

774

775

776

777

778

779

780

781

782

783

784

785

786

787

788

789

790

791

792

793

794

795

796

797

798

799

800

801

802

803

804

805

806

sensor devices in an auditory attention detection task. In: Proceedings of the Annual International Conference of the IEEE Engineering in Medicine and Biology Society, EMBS. Institute of Electrical and Electronics Engineers Inc., 77-80. DOI: 10.1109/EMBC.2018.8512212.

Nielsen M. Neural Networks and Deep Learning.

Nossier SA, Rizk MRM, Moussa ND, el Shehaby S. 2019. Enhanced smart hearing aid using deep neural networks. Alexandria Engineering Journal 58:539-550. DOI: 10.1016/j.aej.2019.05.006.

Pan H, Pang Z, Wang Y, Wang Y, Chen L. 2020. A New Image Recognition and Classification Method Combining Transfer Learning Algorithm and MobileNet Model for Welding Defects. IEEE Access 8:119951-119960. DOI: 10.1109/ACCESS.2020.3005450.

Radio books for children. Available at http://www.radioboeken.eu/kinderradioboeken.php?lang $=N L$ (accessed March 27, 2021).

Rashid M, Bari BS, Hasan MJ, Razman MAM, Musa RM, Nasir AFA, Majeed APPA. 2021. The classification of motor imagery response: an accuracy enhancement through the ensemble of random subspace k-NN. PeerJ Computer Science 7:1-31. DOI: 10.7717/peerj-cs.374.

Ravi D, Wong C, Deligianni F, Berthelot M, Andreu-Perez J, Lo B, Yang GZ. 2017. Deep Learning for Health Informatics. IEEE Journal of Biomedical and Health Informatics 21:421. DOI: 10.1109/JBHI.2016.2636665.

Sanjay HS, Hiremath B V., Prithvi BS, Dinesh PA. 2020. Machine learning based assessment of auditory threshold perception in human beings. SN Applied Sciences 2:1-10. DOI: 10.1007/s42452-019-1929-7.

Sermanet P, Eigen D, Zhang X, Mathieu M, Fergus R, LeCun Y. 2013. OverFeat: Integrated Recognition, Localization and Detection using Convolutional Networks.

Shao S, McAleer S, Yan R, Baldi P. 2019. Highly Accurate Machine Fault Diagnosis Using Deep Transfer Learning. IEEE Transactions on Industrial Informatics 15:2446-2455. DOI: 10.1109/TII.2018.2864759.

Shirzhiyan Z, Shamsi E, Jafarpisheh AS, Jafari AH. 2019. Objective classification of auditory brainstem responses to consonant-vowel syllables using local discriminant bases. Speech Communication 114:36-48. DOI: 10.1016/j.specom.2019.09.003.

Simonyan K, Zisserman A. 2015. Very deep convolutional networks for large-scale image recognition. In: 3rd International Conference on Learning Representations, ICLR 2015 Conference Track Proceedings. International Conference on Learning Representations, ICLR,.

Sriraam N. 2012. EEG based automated detection of auditory loss: A pilot study. Expert Systems with Applications 39:723-731. DOI: 10.1016/j.eswa.2011.07.064.

Tan L, Chen Y, Maloney TC, Caré MM, Holland SK, Lu LJ. 2013. Combined analysis of sMRI and fMRI imaging data provides accurate disease markers for hearing impairment. NeuroImage: Clinical 3:416-428. DOI: 10.1016/j.nicl.2013.09.008.

Tang C, Lee E. 2019. Hearing loss identification via wavelet entropy and combination of Tabu search and particle swarm optimization. In: International Conference on Digital Signal Processing, DSP. Institute of Electrical and Electronics Engineers Inc.,. DOI: 10.1109/ICDSP.2018.8631839.

Thorpe B, Dussard T. 2018. Classification of Speech Using MATLAB and K-Nearest Neighbour Model: Aid to the Hearing Impaired. In: Conference Proceedings - IEEE SOUTHEASTCON. Institute of Electrical and Electronics Engineers Inc.,. DOI: 10.1109/SECON.2018.8479223.

Peer] Comput. Sci. reviewing PDF | (CS-2021:03:59631:1:0:NEW 6 Jun 2021) 
807 Xue P, Bai J, Wang Q, Zhang X, Feng P. 2018. Analysis and classification of the nasal finals in 808 hearing-impaired patients using tongue movement features. Speech Communication 104:57809 65. DOI: 10.1016/j.specom.2018.09.008.

810 Yan R, Gao RX, Chen X. 2014. Wavelets for fault diagnosis of rotary machines: A review with 811 applications. Signal Processing 96:1-15. DOI: 10.1016/j.sigpro.2013.04.015.

812 Zhang R, McAllister G, Scotney B, McClean S, Houston G. 2006. Combining wavelet analysis 813 and Bayesian networks for the classification of auditory brainstem response. IEEE Transactions on Information Technology in Biomedicine 10:458-467. DOI: 10.1109/TITB.2005.863865. 


\section{Figure 1}

The overall procedure of hearing deficiency diagnosis method.

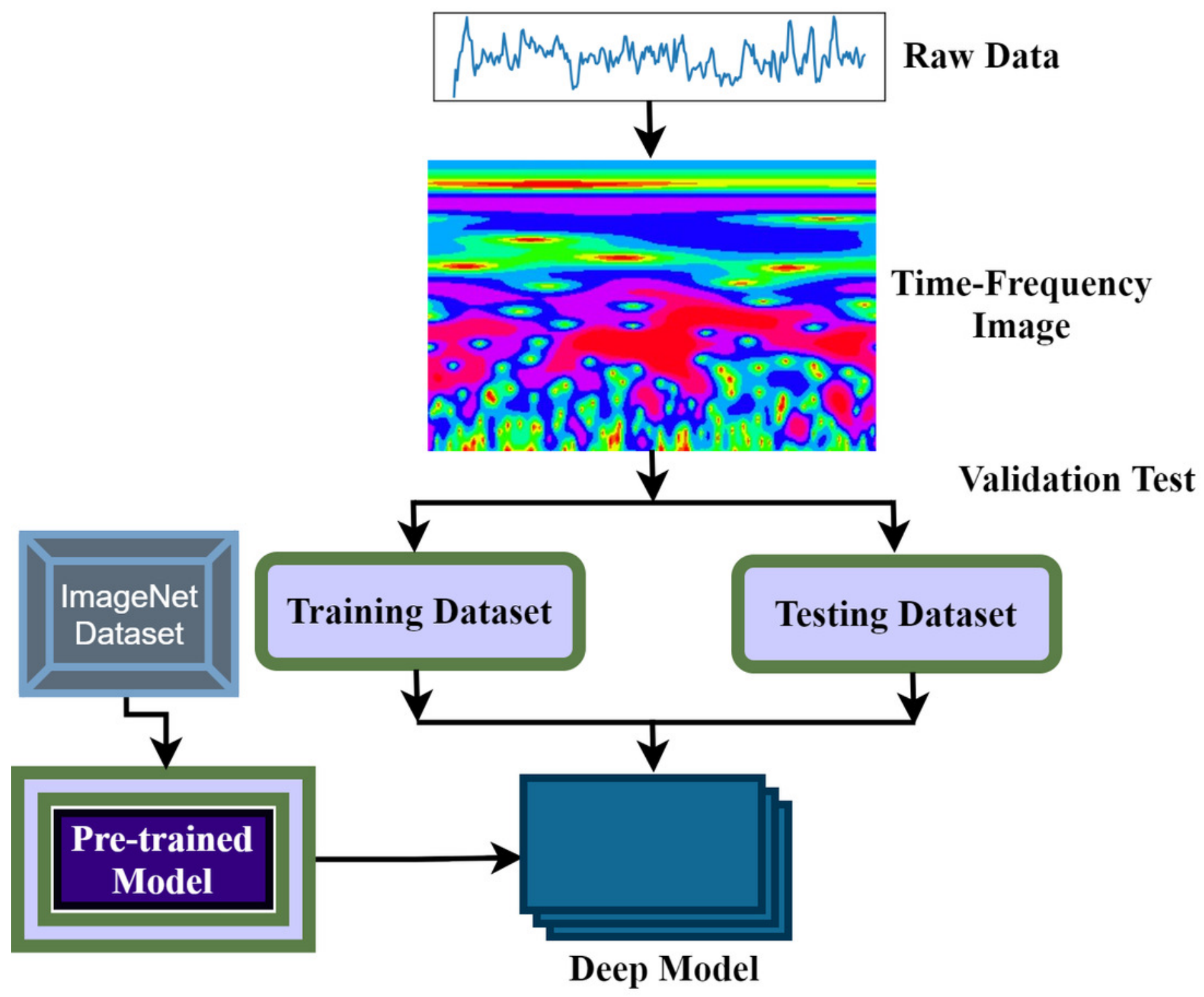


Figure 2

AEP raw data plotting in $2 \mathrm{~s}$ decision window: $(A)$ hear auditory stimulus with the left ear (B) hear auditory stimulus with the right ear.

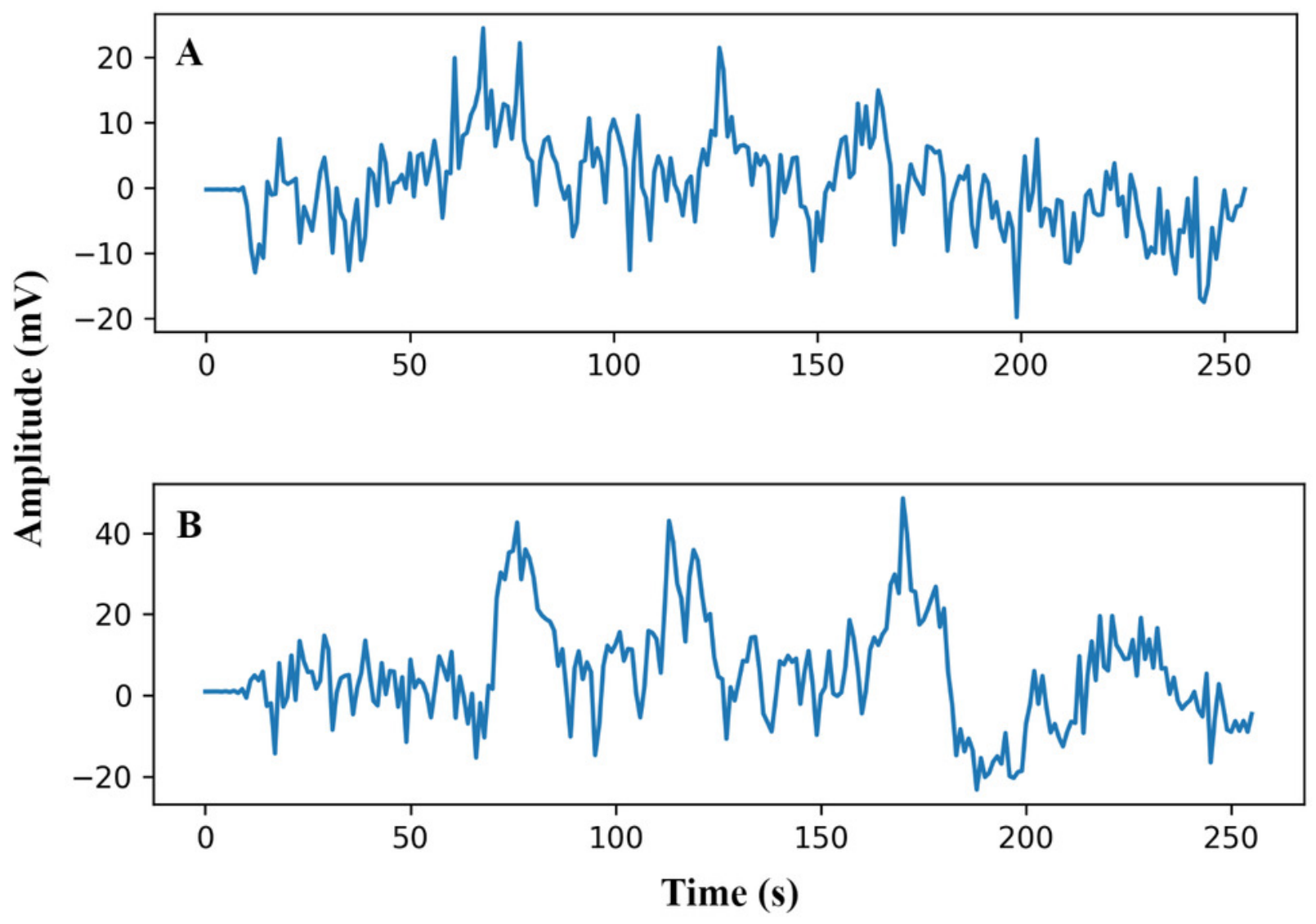


Figure 3

The Transformation process from time-domain signal to time-frequency domain image.

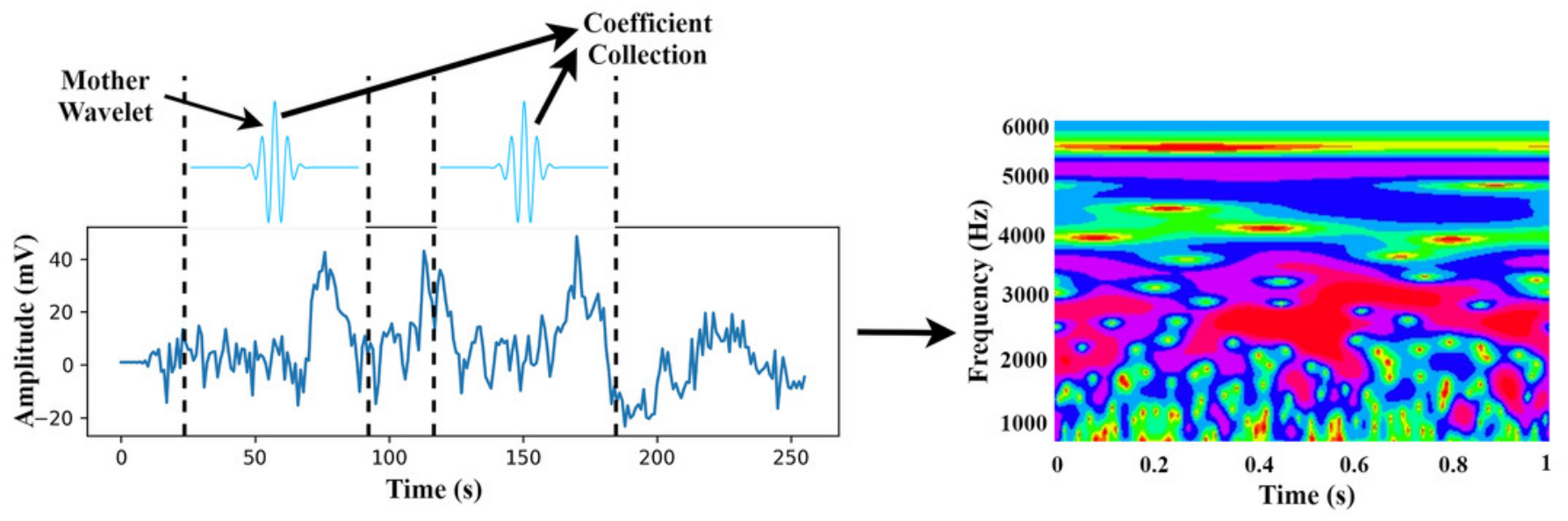




\section{Figure 4}

The time-frequency image of 64 channels data.

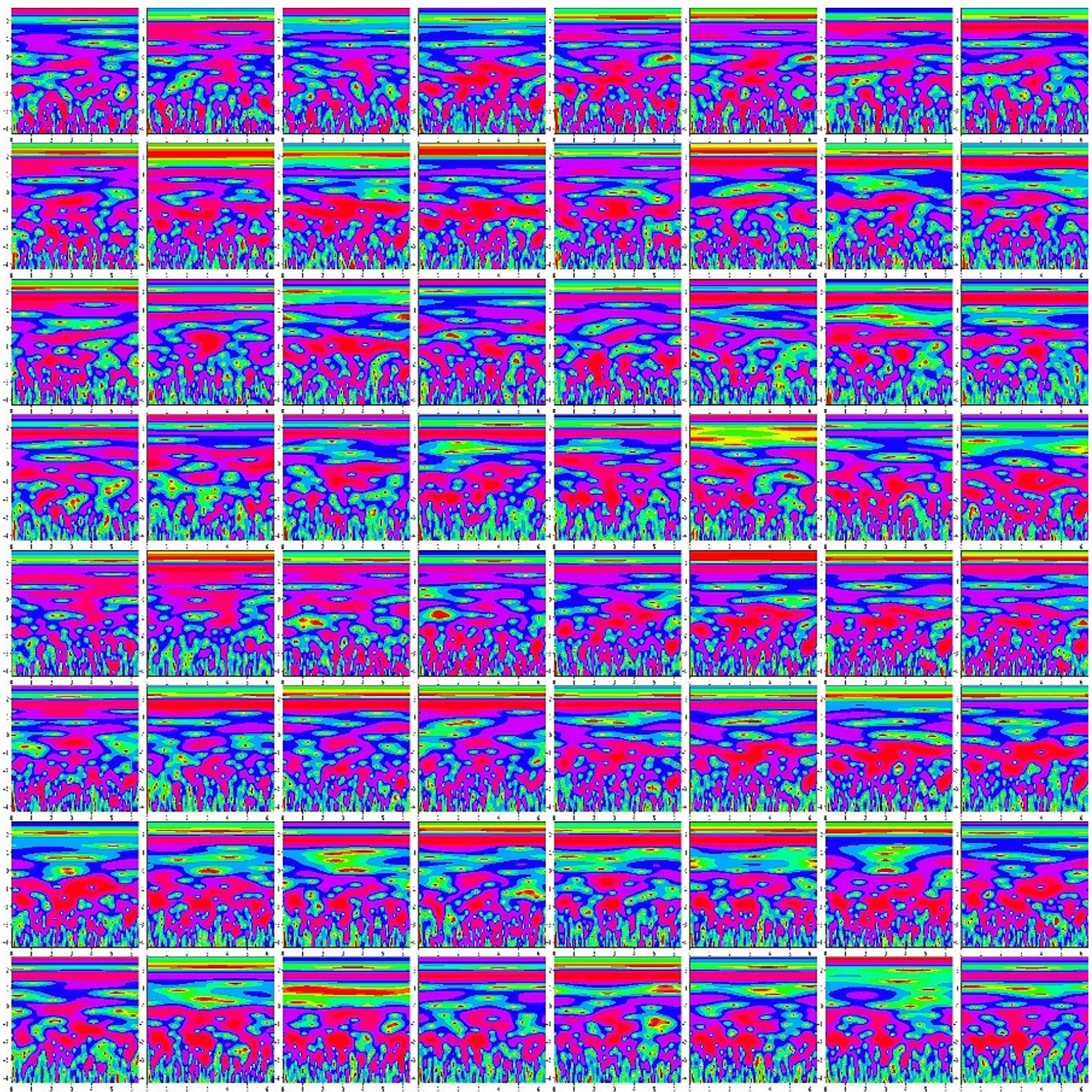


Figure 5

Typical Convolutional Neural Network Architecture.

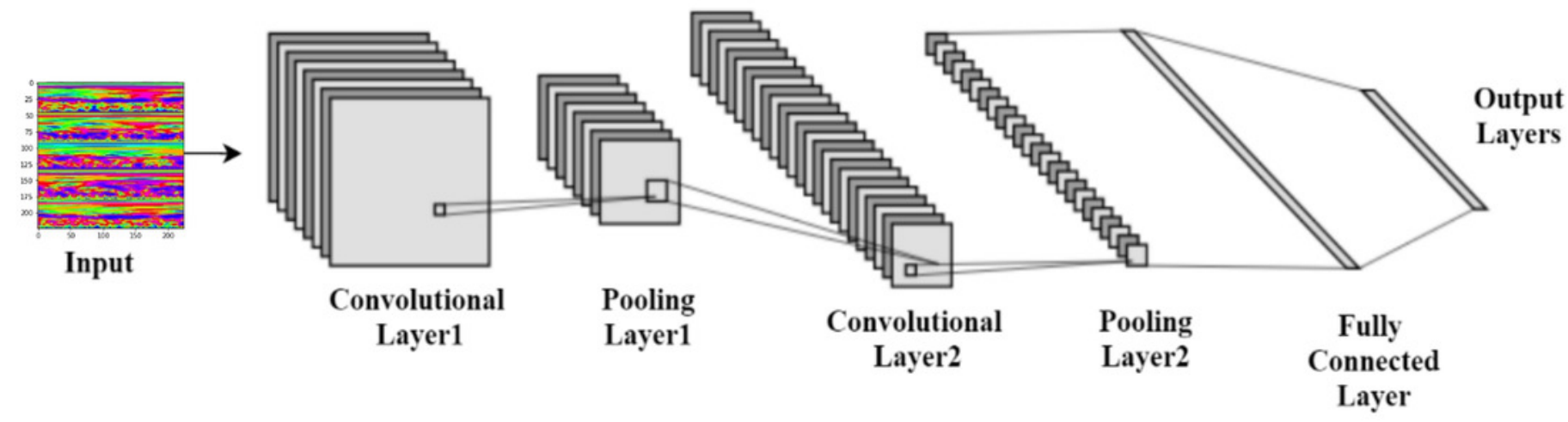


Figure 6

Transfer Learning Procedure of the Proposed Method. 


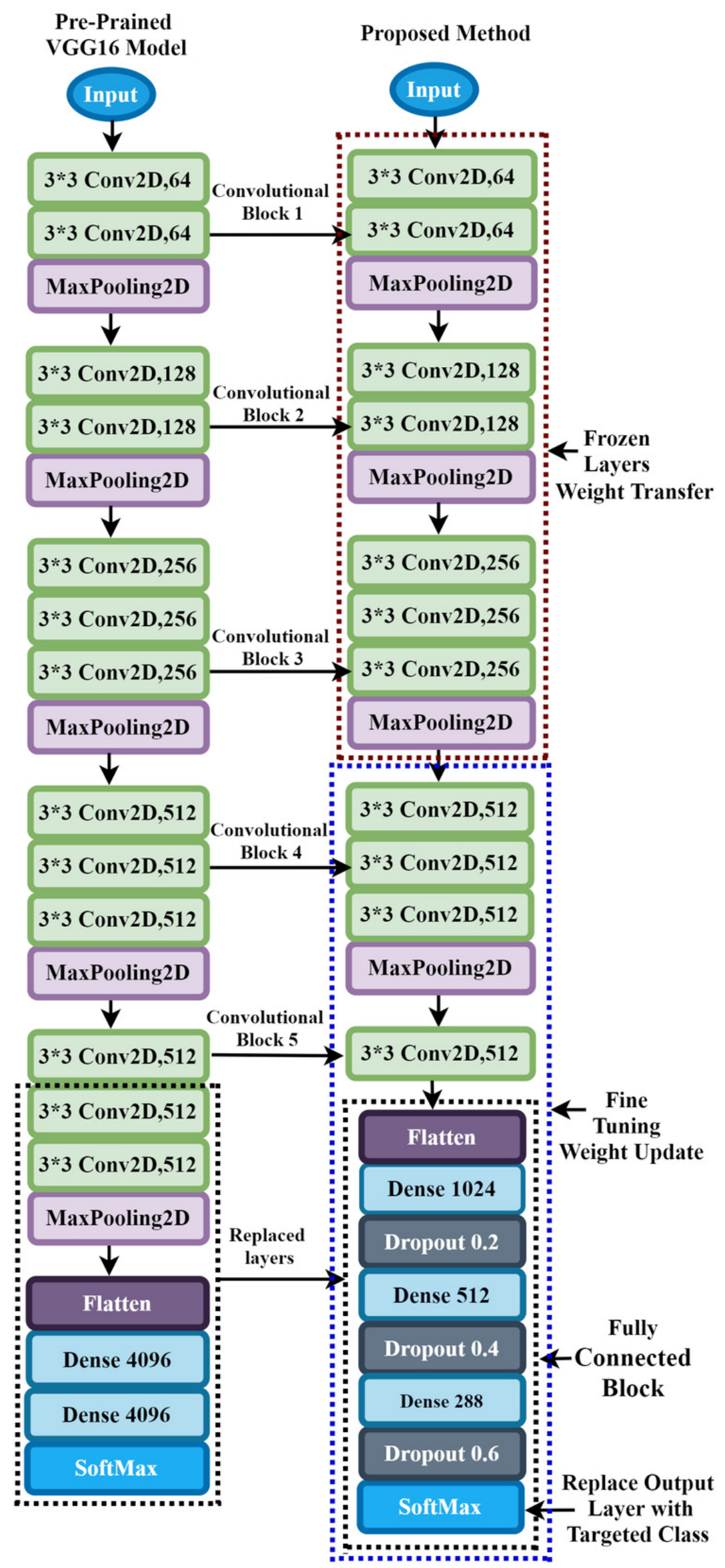


Figure 7

The overall accuracy and loss curve of the proposed TL method for 1s decision window.

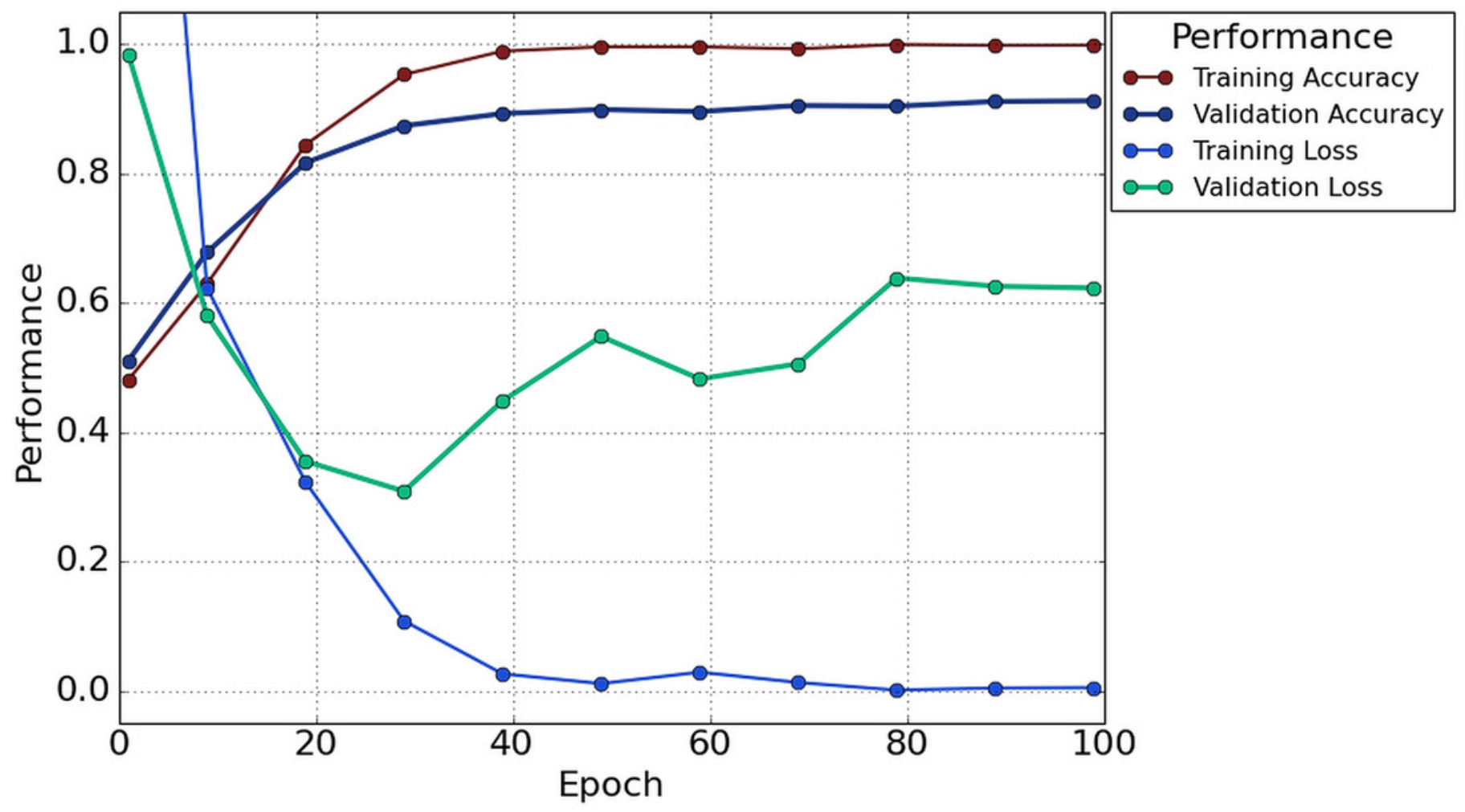


Figure 8

The overall accuracy and loss curve of the proposed TL method for $2 \mathrm{~s}$ decision window.

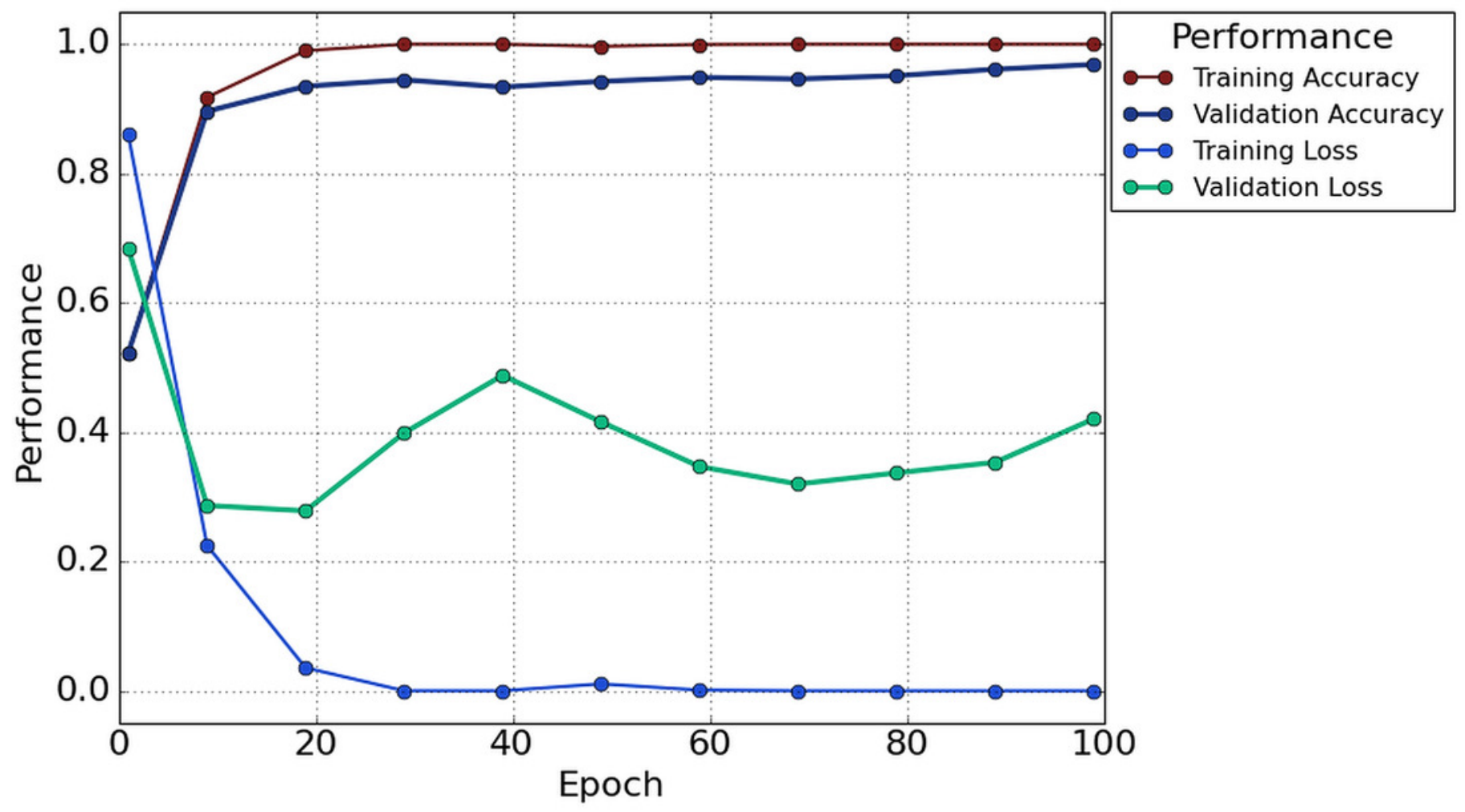




\section{Figure 9}

Confusion matrix for 1s decision windows (A)subject1 (B)subject2 (C)subject3 (D)subject4 (E)subject5 (F)subject6 (G)subject7 (H)subject8 (I)subject9 (J)subject10 (K)subject11 (L)subject12 (M)subject13 (N)subject14 (O)subject15 (P)subject16

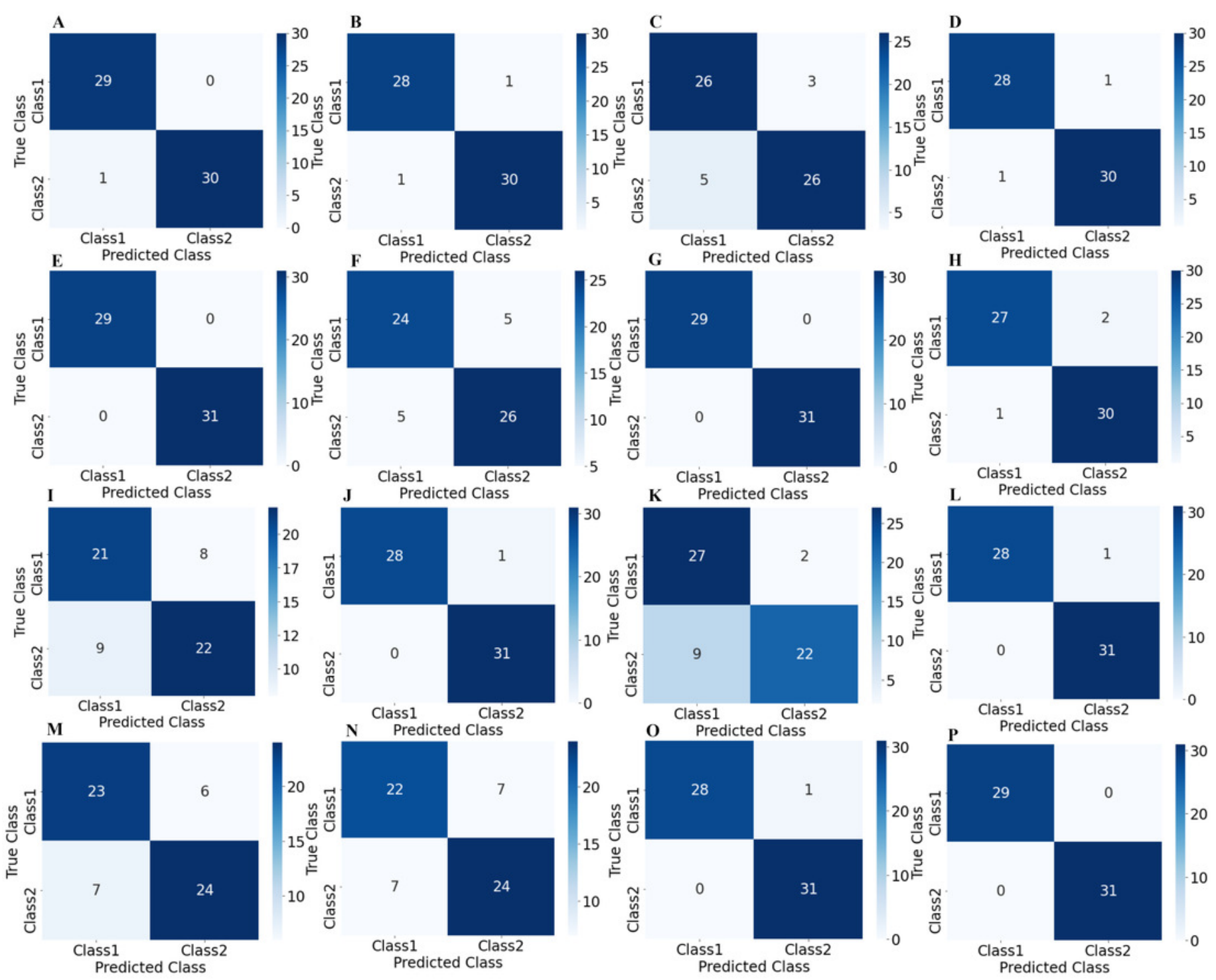


Figure 10

Confusion matrix for $2 \mathrm{~s}$ decision windows $(\mathrm{A})$ subject1 (B)subject2 (C)subject3 (D)subject4 (E)subject5 (F)subject6 (G)subject7 (H)subject8 (I)subject9 (J)subject10 (K)subject11 (L)subject12 (M)subject13 (N)subject14 (O)subject15 (P)subject16
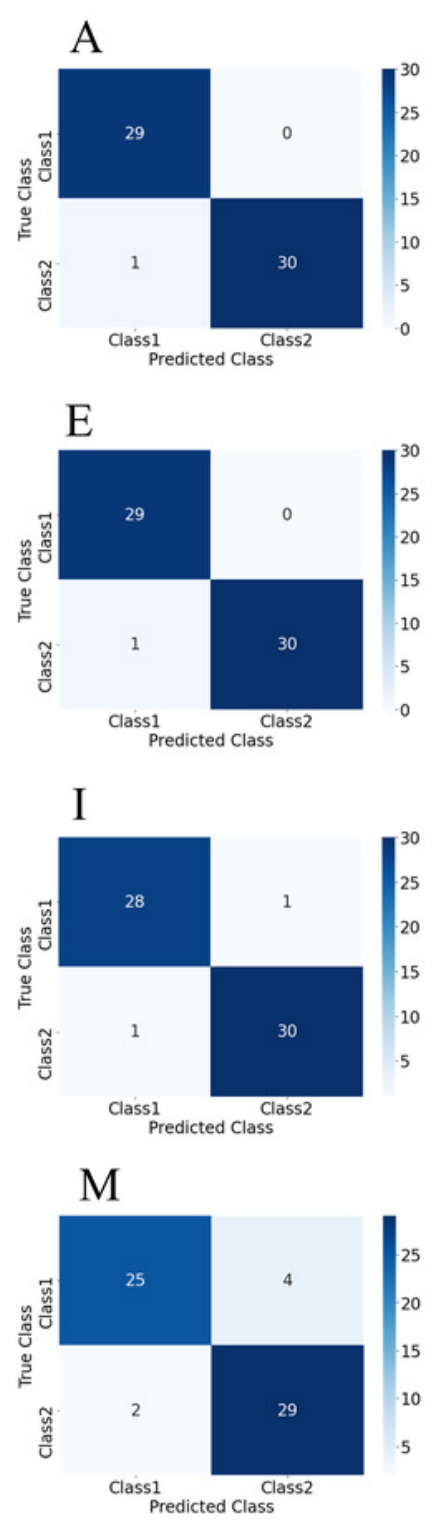
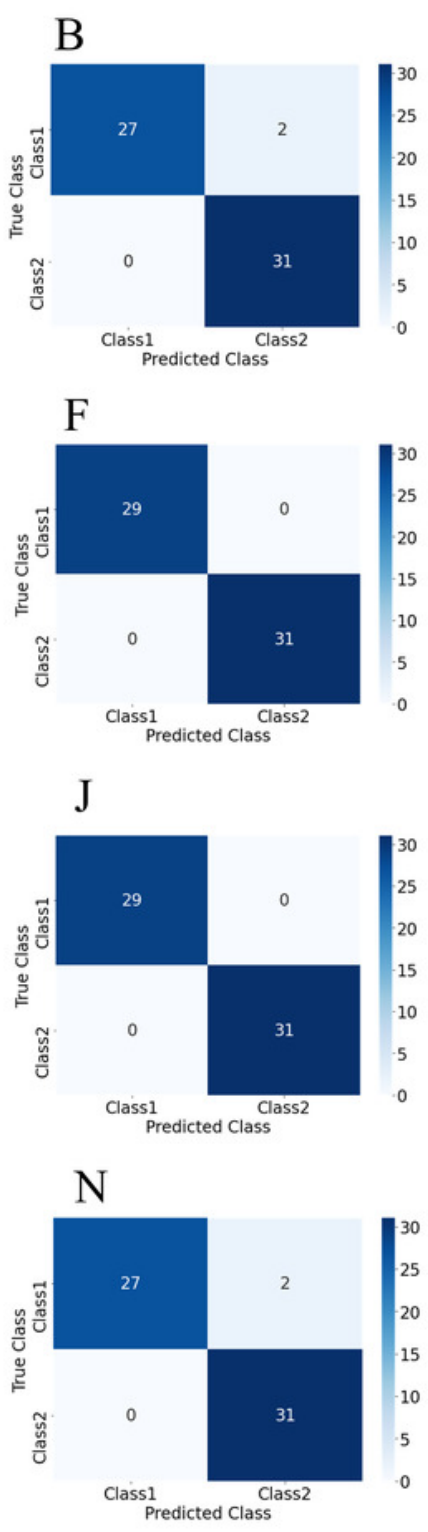
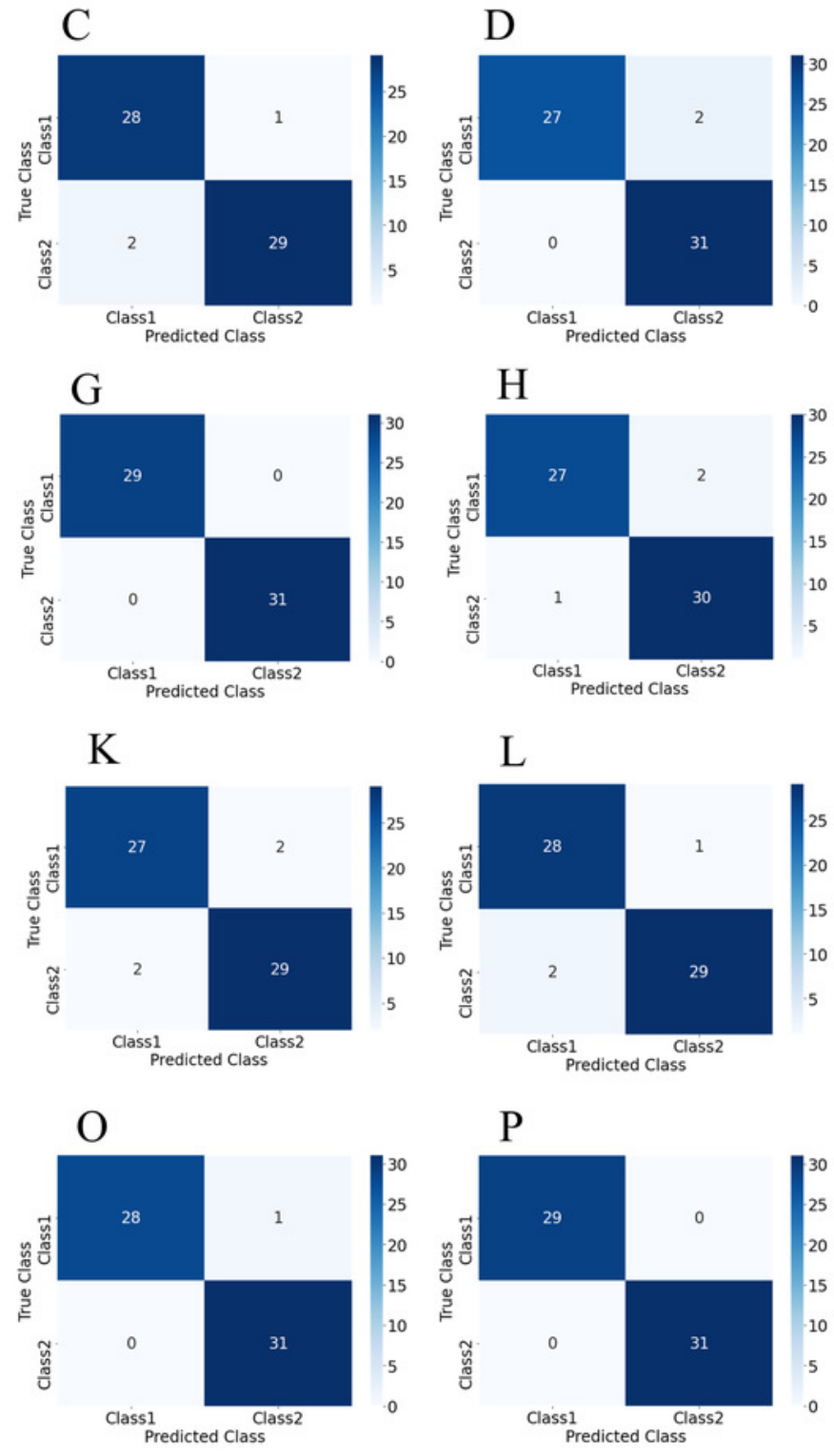


\section{Figure 11}

Hearing deficiency detection performance of the proposed TL architecture for two different window lengths.

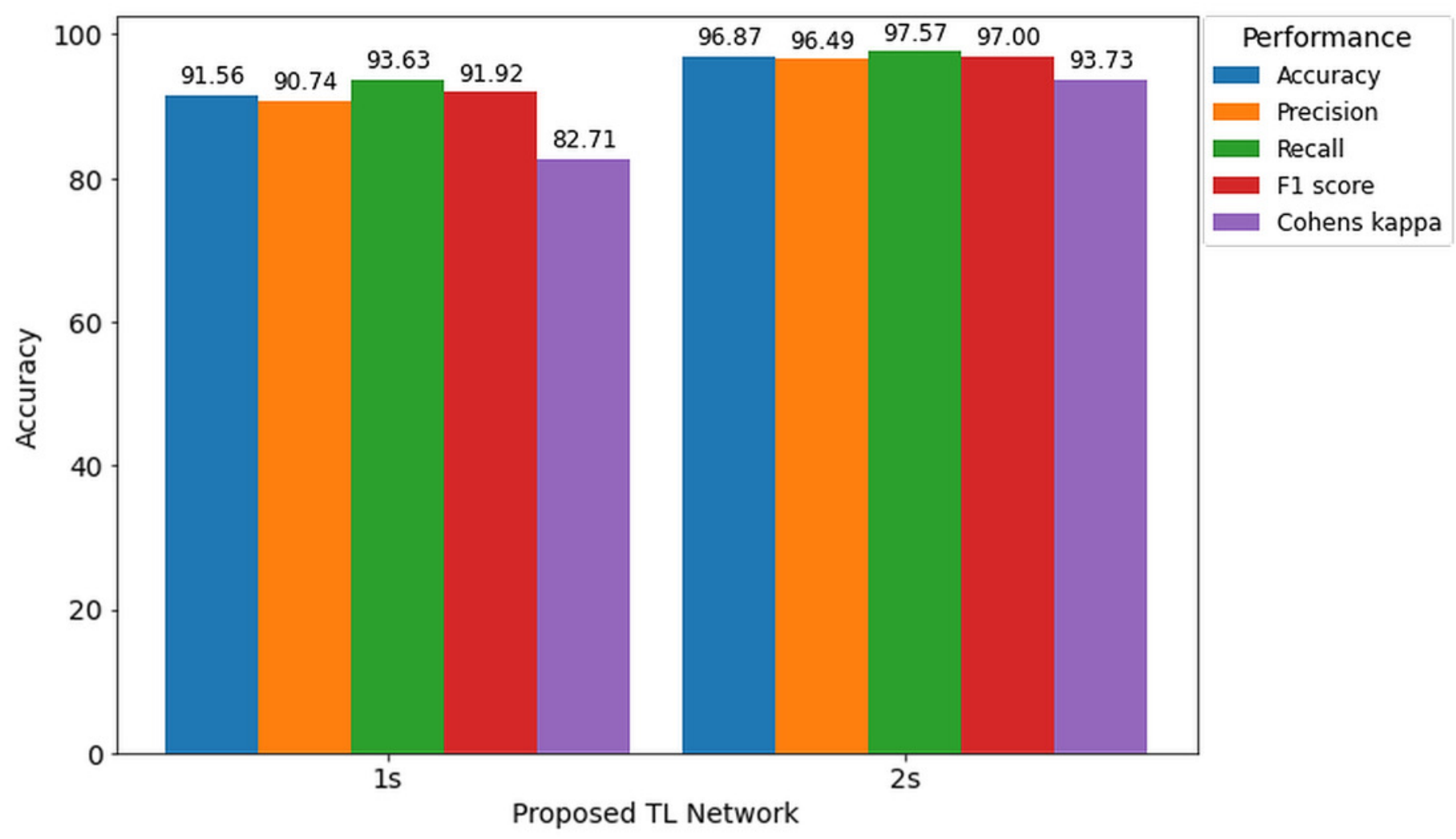


Figure 12

The performance comparison with other pre-trained architectures.

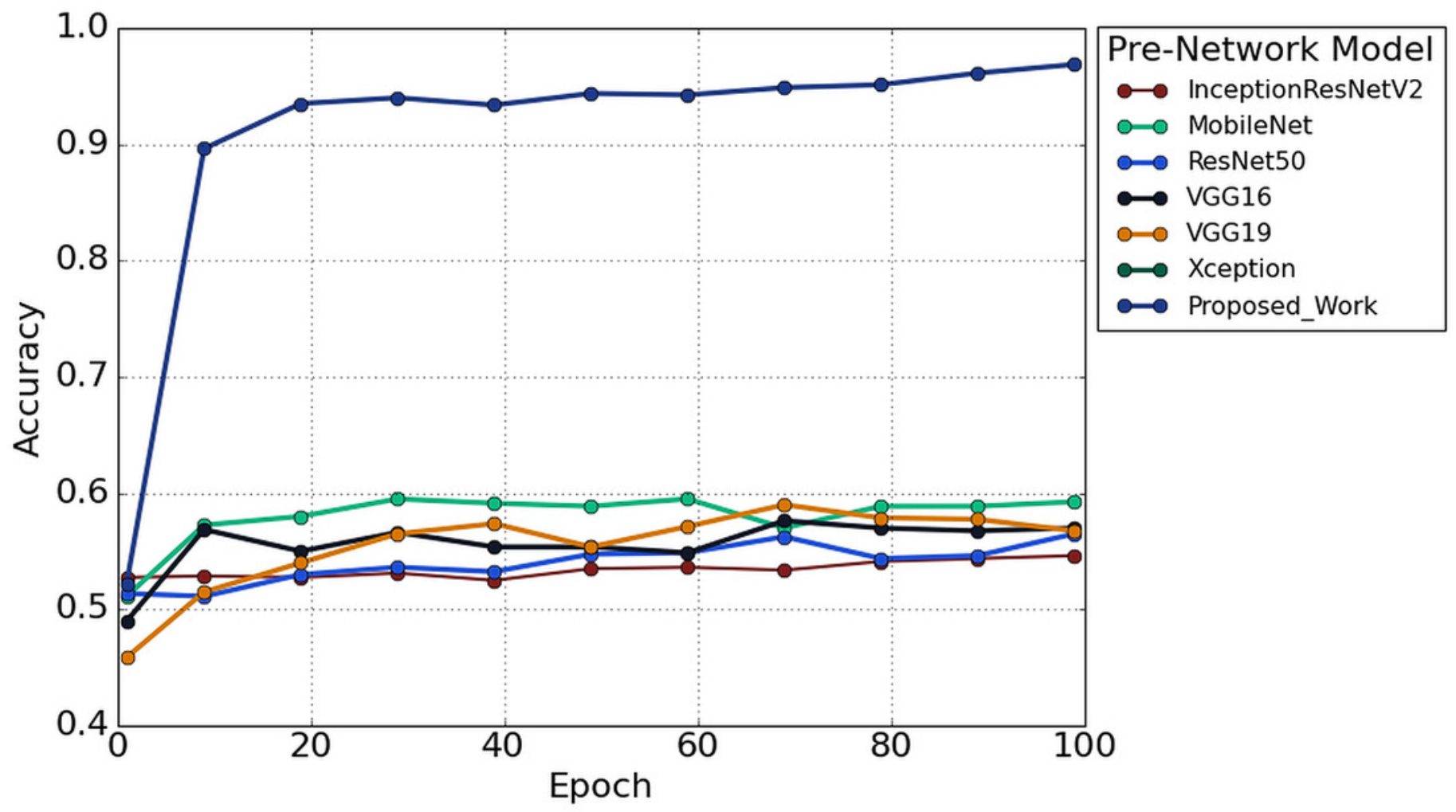




\section{Box 1 (on next page)}

Training procedure of proposed TL architecture. 
1 Box 1: Training procedure of proposed TL architecture.

\begin{tabular}{|l|l|}
\hline \multicolumn{2}{|l|}{ The training steps of the proposed TL architecture: } \\
\hline Step1: & Load the VGG16 base model with the pre-trained weights. \\
\hline Step 2: & $\begin{array}{l}\text { Freeze some layers in the base model by setting trainable }=\text { False. In the non- } \\
\text { trainable layers, the weights will not train. }\end{array}$ \\
\hline Step 3: & $\begin{array}{l}\text { Create a new model by replacing some layers of VGG16 with new layers and re- } \\
\text { train the layers with the layers where the trainable }=\text { True. }\end{array}$ \\
\hline Step 4: & Train the new model with the dataset. \\
\hline
\end{tabular}

2 


\section{Table 1 (on next page)}

Parameter of proposed TL architecture. 
1 Table 1: Parameter of proposed TL architecture.

\begin{tabular}{|c|c|c|}
\hline Layer (type) & Output & $\begin{array}{l}\text { Number of } \\
\text { parameters }\end{array}$ \\
\hline Input & $224 * 224 * 3$ & 0 \\
\hline Block1-Conv2D & $224 * 224 * 64$ & 1792 \\
\hline Block1-Conv2D & $224 * 224 * 64$ & 36928 \\
\hline Block1-MaxPooling2D & $112 * 112 * 64$ & 0 \\
\hline Block2-Conv2D & $112 * 112 * 128$ & 73856 \\
\hline Block2-Conv2D & $112 * 112 * 128$ & 147584 \\
\hline Block2-MaxPooling2D & $56 * 56 * 128$ & 0 \\
\hline Block3-Conv2D & $56 * 56 * 256$ & 295168 \\
\hline Block3-Conv2D & $56 * 56 * 256$ & 590080 \\
\hline Block3-Conv2D & $56 * 56 * 256$ & 590080 \\
\hline Block3-MaxPooling2D & $28 * 28 * 256$ & 0 \\
\hline Block4-Conv2D & $28 * 28 * 512$ & 1180160 \\
\hline Block4-Conv2D & $28 * 28 * 512$ & 2359808 \\
\hline Block4-Conv2D & $28 * 28 * 512$ & 2359808 \\
\hline Block4-MaxPooling2D & $14 * 14 * 512$ & 0 \\
\hline Block5-Conv2D & $14 * 14 * 512$ & 2359808 \\
\hline Flatten-Flatten & $1 * 1 * 100352$ & 0 \\
\hline fc1-Dense & $1 * 1 * 1024$ & 102761472 \\
\hline dropout-Dropout & $1 * 1 * 1024$ & 0 \\
\hline fc2-Dense & $1 * 1 * 512$ & 524800 \\
\hline dropout_1-Dropout & $1 * 1 * 512$ & 0 \\
\hline Fc3-Dense & $1 * 1 * 288$ & 147744 \\
\hline dropout_2-Dropout & $1 * 1 * 288$ & 0 \\
\hline Output-Dense & $\mathrm{C}$ & $288 * \mathrm{C}+\mathrm{C}$ \\
\hline
\end{tabular}




\section{Table 2 (on next page)}

Performance of proposed model for 1s decision window. 
1 Table 2. Performance of proposed model for $1 \mathrm{~s}$ decision window.

\begin{tabular}{|c|c|c|c|c|c|}
\hline Subject & Accuracy & Precision & Recall & F1 Score & $\begin{array}{c}\text { Cohens } \\
\text { Kappa }\end{array}$ \\
\hline Subject-1 & 0.9833 & 0.9688 & 1.0 & 0.9841 & 0.9666 \\
\hline Subject-2 & 0.9667 & 1.0 & 0.9355 & 0.96667 & 0.9334 \\
\hline Subject-3 & 0.8667 & 0.8108 & 0.9677 & 0.8824 & 0.7312 \\
\hline Subject-4 & 0.9667 & 0.9393 & 1.0 & 0.9688 & 0.9331 \\
\hline Subject-5 & 1.0 & 1.0 & 1.0 & 1.0 & 1.0 \\
\hline Subject-6 & 0.8333 & 0.8387 & 0.8387 & 0.8387 & 0.6663 \\
\hline Subject-7 & 1.0 & 1.0 & 1.0 & 1.0 & 1.0 \\
\hline Subject-8 & 0.95 & 0.9667 & 0.9355 & 0.9508 & 0.9 \\
\hline Subject-9 & 0.7667 & 0.7167 & 0.7933 & 0.7367 & 0.4833 \\
\hline Subject-10 & 0.9833 & 1.0 & 0.9677 & 0.9836 & 0.9667 \\
\hline Subject-11 & 0.8167 & 0.7409 & 1.0 & 0.8578 & 0.6241 \\
\hline Subject-12 & 0.9833 & 1.0 & 0.9632 & 0.9853 & 0.9567 \\
\hline Subject-13 & 0.7833 & 0.8214 & 0.7419 & 0.7797 & 0.5676 \\
\hline Subject-14 & 0.76667 & 0.7453 & 0.8365 & 0.7892 & 0.5378 \\
\hline Subject-15 & 0.9833 & 0.9688 & 1.0 & 0.9841 & 0.9666 \\
\hline Subject-16 & 1.0 & 1.0 & 1.0 & 1.0 & 1.0 \\
\hline Average & $91.56 \% \pm$ & $90.74 \% \pm$ & $93.63 \% \pm$ & $91.92 \% \pm$ & $82.71 \% \pm$ \\
\pm SD & $8.91 \%$ & $10.47 \%$ & $8.25 \%$ & $8.79 \%$ & $18.34 \%$ \\
\hline
\end{tabular}

2 


\section{Table 3(on next page)}

Performance of proposed model for $2 \mathrm{~s}$ decision window. 
1 Table 3: Performance of proposed model for 2s decision window.

\begin{tabular}{|l|l|l|l|l|l|}
\hline Subject & Accuracy & Precision & Recall & F1 Score & $\begin{array}{l}\text { Cohens } \\
\text { Kappa }\end{array}$ \\
\hline Subject-1 & 0.9833 & 1.0 & 0.9677 & 0.9836 & 0.9666 \\
\hline Subject-2 & 0.9666 & 0.9393 & 1.0 & 0.9687 & 0.9331 \\
\hline Subject-3 & 0.95 & 0.9666 & 0.9354 & 0.9508 & 0.9 \\
\hline Subject-4 & 0.9666 & 0.9393 & 1.0 & 0.96875 & 0.9331 \\
\hline Subject-5 & 0.9833 & 1.0 & 0.9677 & 0.9836 & 0.9666 \\
\hline Subject-6 & 1.0 & 1.0 & 1.0 & 1.0 & 1.0 \\
\hline Subject-7 & 1.0 & 1.0 & 1.0 & 1.0 & 1.0 \\
\hline Subject-8 & 0.95 & 0.9375 & 0.9677 & 0.9523 & 0.8997 \\
\hline Subject-9 & 0.9666 & 0.9677 & 0.9677 & 0.9677 & 0.9332 \\
\hline Subject-10 & 1.0 & 1.0 & 1.0 & 1.0 & 1.0 \\
\hline Subject-11 & 0.9333 & 0.9354 & 0.9354 & 0.9354 & 0.8665 \\
\hline Subject-12 & 0.95 & 0.9666 & 0.9354 & 0.9508 & 0.9 \\
\hline Subject-13 & 0.9 & 0.8787 & 0.9354 & 0.9062 & 0.7993 \\
\hline Subject-14 & 0.9666 & 0.9393 & 1.0 & 0.9687 & 0.9331 \\
\hline Subject-15 & 0.9833 & 0.9687 & 1.0 & 0.9841 & 0.9665 \\
\hline Subject-16 & 1.0 & 1.0 & 1.0 & 1.0 & 1.0 \\
\hline $\begin{array}{l}\text { Average } \\
\pm \text { SD }\end{array}$ & $96.87 \% \pm$ & $96.49 \% \pm$ & $97.57 \% \pm$ & $97 \% \pm$ & $93.73 \% \pm$ \\
\hline
\end{tabular}

2 


\section{Table 4 (on next page)}

Performance comparison with six popular TL models. 
1 Table 4: Performance comparison with six popular TL models.

\begin{tabular}{|c|c|c|c|c|c|}
\hline $\begin{array}{c}\text { Pre-network } \\
\text { Model }\end{array}$ & $\begin{array}{c}\text { Input } \\
\text { Size }\end{array}$ & $\begin{array}{c}\text { Trainable } \\
\text { Parameters }\end{array}$ & $\begin{array}{c}\text { Non- } \\
\text { trainable } \\
\text { Parameters }\end{array}$ & $\begin{array}{c}\text { Total } \\
\text { Parameters }\end{array}$ & $\begin{array}{c}\text { Recognition } \\
\text { Accuracy (\%) }\end{array}$ \\
\hline VGG16 & 224 & 8,194 & $134,260,544$ & $134,268,738$ & 57.375 \\
\hline InceptionResNetV2 & 224 & 3,074 & $54,336,736$ & $54,339,810$ & 54.000 \\
\hline ResNet50 & 224 & 4,098 & $23,587,712$ & $23,591,810$ & 54.875 \\
\hline MobileNet & 224 & 2,002 & $4,253,864$ & $4,255,866$ & 60.250 \\
\hline Xception & 224 & 4,098 & $20,861,480$ & $20,865,578$ & 57.625 \\
\hline VGG19 & 224 & 8,194 & $139,570,240$ & $139,578,434$ & 56.625 \\
\hline Proposed Model & $\mathbf{2 2 4}$ & $\mathbf{1 0 3 , 4 3 4 , 5 9 4}$ & $\mathbf{9 , 9 9 5 , 0 7 2}$ & $\mathbf{1 1 3 , 4 2 9 , 6 6 6}$ & $\mathbf{9 6 . 8 7} \pm \mathbf{2 . 7 8}$ \\
\hline
\end{tabular}

2 


\section{Table 5 (on next page)}

Performance comparison of related AEP studies. 
1 Table 5: Performance comparison of related AEP studies.

2

\begin{tabular}{|c|c|c|c|c|c|c|}
\hline \multirow[t]{2}{*}{ Reference } & \multirow[t]{2}{*}{ Year } & \multicolumn{2}{|l|}{ Data } & \multirow{2}{*}{$\begin{array}{c}\text { Feature } \\
\text { Extraction }\end{array}$} & \multirow{2}{*}{$\begin{array}{c}\text { Classification } \\
\text { Method }\end{array}$} & \multirow{2}{*}{$\begin{array}{c}\text { Classification } \\
\text { Accuracy } \\
(\%)\end{array}$} \\
\hline & & Subject & Class & & & \\
\hline$[15]$ & 2018 & 180 & 2 & WE & TS-PSO & 86.17 \\
\hline [32] & 2018 & 32 & 2 & $\begin{array}{l}\text { Global and } \\
\text { nodal graph }\end{array}$ & SVM & 85.71 \\
\hline [14] & 2004 & 200 & 3 & WPT & SVM & 74.7 \\
\hline [18] & 2006 & 8 & 2 & DWT & $\begin{array}{c}\text { Bayesian } \\
\text { network } \\
\text { classification }\end{array}$ & 78.80 \\
\hline [33] & 2013 & 39 & 2 & SIFT & SVM & 87 \\
\hline [19] & 2019 & $\begin{array}{c}\text { Observation: } \\
671\end{array}$ & 2 & FFT & SVM & 78.7 \\
\hline [34] & 2019 & Observation:671 & 2 & Raw AEP & $\mathrm{CNN}$ & 94.1 \\
\hline [35] & 2016 & $\begin{array}{l}\text { Observation: } \\
280 \\
\text { Subjects: } 151\end{array}$ & 2 & $\begin{array}{l}\text { latency, } \\
\text { FFT and } \\
\text { DWT }\end{array}$ & $\begin{array}{c}\text { A feed- } \\
\text { forward } \\
\text { multilayer } \\
\text { perceptron }\end{array}$ & 90.74 \\
\hline $\begin{array}{l}\text { Proposed } \\
\text { Work }\end{array}$ & - & $\begin{array}{c}\text { Observations: } \\
3200\end{array}$ & 2 & CWT & $\begin{array}{l}\text { Improved- } \\
\text { VGG16 }\end{array}$ & 96.87 \\
\hline
\end{tabular}

3 